\title{
Del Pezzo surfaces with infinite automorphism groups
}

\author{
Ivan Cheltsov and Yuri Prokhorov
}

\begin{abstract}
We classify del Pezzo surfaces with Du Val singularities that have infinite automorphism groups and describe the connected components of their automorphisms groups.
\end{abstract}

\section{Introduction}

Throughout this paper, we always assume that all varieties are projective and defined over an algebraically closed field $\mathbb{k}$ of characteristic 0 .

Automorphism groups of smooth del Pezzo surfaces are well studied (see, for example, [Dol12, DI09]). In particular, if $X$ is a smooth del Pezzo surface, then $\operatorname{Aut}(X)$ is infinite if and only if $X$ is toric. Moreover, if $X$ is a smooth toric del Pezzo surface, then $\operatorname{Aut}^{0}(X)$ can be described as follows:

\begin{tabular}{|c|c|c|c|}
\hline$K_{X}^{2}$ & $\operatorname{Aut}^{0}(X)$ & \multicolumn{2}{|c|}{ Equation \& total space } \\
\hline 6 & $\mathbb{G}_{\mathrm{m}}^{2}$ & $u_{0} v_{0} w_{0}=u_{1} v_{1} w_{1}$ & $\mathbb{P}^{1} \times \mathbb{P}^{1} \times \mathbb{P}^{1}$ \\
\hline 7 & $\mathbb{B}_{2} \times \mathbb{B}_{2}$ & & \\
\hline 8 & $\mathbb{G}_{\mathrm{a}}^{2} \rtimes \mathrm{GL}_{2}(\mathbb{k})$ & $u_{0} v_{0}=u_{1} v_{1}$ & $\mathbb{P}^{2} \times \mathbb{P}^{1}$ \\
\hline 8 & $\mathrm{PGL}_{2}(\mathbb{k}) \times \mathrm{PGL}_{2}(\mathbb{k})$ & - & $\mathbb{P}^{1} \times \mathbb{P}^{1}$ \\
\hline 9 & $\mathrm{PGL}_{3}(\mathbb{k})$ & - & $\mathbb{P}^{2}$ \\
\hline
\end{tabular}

where $\mathbb{G}_{\mathrm{a}}$ is a one-dimensional unipotent additive group, $\mathbb{G}_{\mathrm{m}}$ is a one-dimensional algebraic torus, and $\mathbb{B}_{2}$ is the Borel subgroup of $\mathrm{PGL}_{2}(\mathbb{k})$. In this paper, we prove similar results for del Pezzo surfaces with at worst Du Val singularities. For short, we call such surfaces $D u$ Val del Pezzo surfaces. Our main result is the following.

Theorem 1.1 (Main theorem). Let $X$ be a Du Val del Pezzo surface. Then the group Aut $(X)$ is infinite if and only if $X$ is described in Table 3 in Section 8.

Everywhere below, the number $n^{0}$ refers to the corresponding surface in Table 3 in Section 8. As a consequence of our classification we have the following.

Corollary 1.2. Let $X$ be a $D u$ Val del Pezzo surface. Then the group $\operatorname{Aut}(X)$ is not reductive if and only if $X$ is one of the 23 surfaces $7^{\circ}, 14^{\circ}, 15^{\circ}, 18^{\circ}, 24^{\circ}, 25^{\circ}, 26^{\circ}, 27^{\circ}, 28^{\circ}, 31^{\circ}, 36^{\circ}, 37^{\circ}$, $38^{\circ}, 39^{\circ}, 42^{\circ}, 43^{\circ}, 44^{\circ}, 45^{\circ}, 46^{\circ}, 48^{\circ}, 49^{\circ}, 50^{\circ}, 51^{\circ}$.

Received 3 August 2020, accepted in final form 4 October 2020.

2020 Mathematics Subject Classification 14J50 (primary), 14J17, 14J26 (secondary).

Keywords: del Pezzo surface, Du Val singularity, automorphism.

This journal is (C) Foundation Compositio Mathematica 2021. This article is distributed with Open Access under the terms of the Creative Commons Attribution Non-Commercial License, which permits non-commercial reuse, distribution, and reproduction in any medium, provided that the original work is properly cited. For commercial re-use, please contact the Foundation Compositio Mathematica.

This work was supported by the Royal Society grant No. IES $\backslash$ R $1 \backslash 180205$ and by the Russian Academic Excellence Project 5-100. 


\section{Cheltsov and Yu. Prokhorov}

Thus, the surfaces listed in this corollary are not $K$-polystable [ABHX21, Mat57], which is known (see [OSS16]).

Corollary 1.3. Let $X$ be a $\mathrm{Du}$ Val del Pezzo surface.

(i) If $K_{X}^{2}=1$ and $\operatorname{Aut}(X)$ is infinite, then $\rho(X)=1$.

(ii) If $K_{X}^{2}>1$ and $\rho(X)=1$, then $\operatorname{Aut}(X)$ is infinite.

(iii) If $K_{X}^{2} \geqslant 6$ or $K_{X}^{2}=5$ and $X$ is singular, then $\operatorname{Aut}(X)$ is infinite.

Many particular parts of our classification have been previously studied from different perspectives. For examples, the Du Val del Pezzo surfaces admitting an effective action of the groups $\mathbb{G}_{\mathrm{a}}^{2}$ and $\mathbb{G}_{\mathrm{a}} \rtimes \mathbb{G}_{\mathrm{m}}$ have been classified in [DL10, DL15]. The classification of toric Du Val del Pezzo surfaces is well known for specialists (see, for example, [PR00]). Du Val del Pezzo surfaces that admit a faithful action of the group $\mathbb{G}_{\mathrm{m}}$ have been studied in [ADHL15, HHS11, Her14] in terms of their Cox rings. Moreover, when we were finishing the final version of this paper, we were informed that Theorem 1.1 has been independently proven in [MS20] using a completely different approach which also works in positive characteristic.

Note that the complete classification of all Du Val del Pezzo surfaces has been known for a long time [DuV34, Dol12]. The basic problem is that it is enormous and that choosing surfaces with infinite automorphism groups typically takes a lot of effort.

Remark 1.4. Almost all surfaces in Table 3 are explicitly given by their defining equations, since they are not always uniquely determined by their degree and singularities. For example, the cubic surface in $\mathbb{P}^{3}$ given by

$$
x_{3} x_{0}^{2}+x_{1}^{3}+x_{2}^{3}+x_{0} x_{1} x_{2}=0
$$

has one singular point of type $\mathrm{D}_{4}$, its automorphism group is finite, and it is not isomorphic to the cubic surface $22^{\circ}$, which has the same singularity. Similarly, the quartic surface in the weighted projective space $\mathbb{P}(1,1,1,2)$ that is given by the equation

$$
y_{2}^{2}=y_{1}^{3} y_{1}^{\prime \prime}+y_{1}^{\prime 4}+y_{1}^{\prime 2} y_{1}^{2}
$$

is a del Pezzo surface of degree 2 that has one singular point of type $\mathrm{E}_{6}$. It is not isomorphic to the del Pezzo surface $11^{\circ}$, which has the same degree and the same singularity. There are more examples like this: the surface $1^{\circ}$ and the sextic surface in $\mathbb{P}(1,1,2,3)$ given by

$$
y_{3}^{2}=y_{2}^{3}+y_{1}^{\prime} y_{1}^{5}+y_{2}^{2} y_{1}^{2}
$$

are the only del Pezzo surfaces of degree 1 with a singular point of type $\mathrm{E}_{8}$. They are not isomorphic. In fact, the latter surface is the only Du Val del Pezzo surface whose class group is $\mathbb{Z}$ that does not appear in our Table 3 (see Remark B.5).

Let us briefly describe the structure of this paper. In Section 2, we present several basic facts about Du Val del Pezzo surfaces which are used in the proof of Theorem 1.1. In Section 3, we prove Theorem 3.8, which together with Theorem 1.1 implies the following.

Corollary 1.5. Let $X$ be a Du Val del Pezzo surface with $K_{X}^{2} \geqslant 3$, and let $\tau(X)$ be its Fano-Weil index. Suppose that $\tau(X)>1$. Then $\operatorname{Aut}(X)$ is infinite.

In Section 4, we prove Theorem 1.1 for del Pezzo surfaces of degree at least 4. Then, in Sections 5, 6, 7, we prove Theorem 1.1 for del Pezzo surfaces of degree 1, 2, 3, respectively. In Section 8, we present Table 3. In Appendix A, we describe lines on del Pezzo surfaces that 


\section{Del Pezzo surfaces}

appear in Table 3 together with the dual graphs of the curves with negative self-intersection numbers on their minimal resolutions. Finally, in Appendix B, we recall the classification of Du Val del Pezzo surfaces whose Weil divisor class group is cyclic and present an alternative proof of Theorem 1.1 for them.

\section{Notation}

Throughout this paper, we use the following notation:

- $\boldsymbol{\mu}_{n}$ is a cyclic subgroup of order $n$.

- $\mathbb{G}_{\mathrm{a}}$ is a one-dimensional unipotent additive group.

- $\mathbb{G}_{\mathrm{m}}$ is a one-dimensional algebraic torus.

- $\mathbb{B}_{n}$ is a Borel subgroup of $\mathrm{PGL}_{n}(\mathbb{k})$.

- $\mathbb{U}_{n}$ is a maximal unipotent subgroup of $\mathrm{PGL}_{n}(\mathbb{k})$.

- $\mathbb{G}_{\mathrm{a}} \rtimes_{(n)} \mathbb{G}_{\mathrm{m}}$ is a semidirect product $\mathbb{G}_{\mathrm{a}}$ and $\mathbb{G}_{\mathrm{m}}$ such that $\mathbb{G}_{\mathrm{m}}$ acts on $\mathbb{G}_{\mathrm{a}}$ as $\mathbf{x} \mapsto t^{n} \mathbf{x}$. This group is isomorphic to the following group:

$$
\left\{\left(\begin{array}{cc}
t^{r} & 0 \\
a & t^{s}
\end{array}\right) \in \mathrm{GL}_{2}(\mathbb{k}) \mid t \in \mathbb{k}^{*} \text { and } a \in \mathbb{k}\right\}
$$

where $n=s-r$. Indeed, the required isomorphism follows from

$$
\left(\begin{array}{cc}
t^{r} & 0 \\
0 & t^{s}
\end{array}\right)\left(\begin{array}{ll}
1 & 0 \\
a & 1
\end{array}\right)\left(\begin{array}{cc}
t^{r} & 0 \\
0 & t^{s}
\end{array}\right)^{-1}=\left(\begin{array}{cc}
1 & 0 \\
a t^{s-r} & 1
\end{array}\right) .
$$

Observe that $\mathbb{G}_{\mathrm{a}} \rtimes_{(0)} \mathbb{G}_{\mathrm{m}}=\mathbb{G}_{\mathrm{a}} \times \mathbb{G}_{\mathrm{m}}, \mathbb{G}_{\mathrm{a}} \rtimes_{(1)} \mathbb{G}_{\mathrm{m}}=\mathbb{B}_{2}$, and

$$
\mathbb{G}_{\mathrm{a}} \rtimes_{(n)} \mathbb{G}_{\mathrm{m}} \cong \mathbb{G}_{\mathrm{a}} \rtimes_{(-n)} \mathbb{G}_{\mathrm{m}} .
$$

Therefore, we will always assume that $n \geqslant 0$. If $n>0$, the center of $\mathbb{G}_{\mathrm{a}} \rtimes_{(n)} \mathbb{G}_{\mathrm{m}}$ is $\boldsymbol{\mu}_{n}$. This implies that $\mathbb{G}_{\mathrm{a}} \rtimes_{\left(n_{1}\right)} \mathbb{G}_{\mathrm{m}} \cong \mathbb{G}_{\mathrm{a}} \rtimes_{\left(n_{2}\right)} \mathbb{G}_{\mathrm{m}} \Longleftrightarrow n_{1}= \pm n_{2}$.

$-\mathbb{F}_{n}$ is the Hirzebruch surface.

- $\mathbb{P}\left(a_{1}, \ldots, a_{n}\right)$ is the weighted projective space.

- For a weighted projective space $\mathbb{P}\left(a_{0}, a_{1}, \ldots, a_{n}\right)$, we denote by $y_{a_{0}}, y_{a_{1}}, \ldots, y_{a_{n}}$ the coordinates on it of weights $a_{0}, a_{1}, \ldots, a_{n}$, respectively.

- For a variety $X$, we denote by $\operatorname{Sing}(X)$ the set of its singular points.

- For a variety $X$, we denote by $\rho(X)$ the rank of the Weil divisor class group $\operatorname{Cl}(X)$.

- For a variety $X$ and its (possibly reducible) reduced subvariety $Y \subseteq X$, we denote by $\operatorname{Aut}(X, Y)$ the group consisting of all automorphisms in $\operatorname{Aut}(X)$ that map $Y$ into itself.

- For a surface $X$ with Du Val singularities, Type $(X)$ denotes the type of its singularities. If Type $(X)=\mathrm{D}_{4} 2 \mathrm{~A}_{1}$, then $\operatorname{Sing}(X)$ consists of a point of type $\mathrm{D}_{4}$ and two points of type $\mathrm{A}_{1}$.

- For a Du Val del Pezzo surface $X$, we denote by $\tau(X)$ its Fano-Weil index, which is defined as follows:

$$
\tau(X)=\max \left\{t \in \mathbb{Z} \mid-K_{X} \sim t A, \text { where } A \text { is a Weil divisor on } X\right\} .
$$




\section{Cheltsov and Yu. Prokhorov}

\section{Del Pezzo surfaces with Du Val singularities}

Let $X$ be a Du Val del Pezzo surface with $d:=K_{X}^{2}$. Then $d$ is known as the degree of the surface $X$. Let $\mu: \widetilde{X} \rightarrow X$ be the minimal resolution of singularities. Then

$$
K_{\tilde{X}} \sim \mu^{*} K_{X}
$$

so that $\widetilde{X}$ is a weak del Pezzo surface, that is, the anticanonical divisor $-K_{\widetilde{X}}$ is nef and big. By the Noether formula, $d=10-\rho(X) \leqslant 9$, and by the genus formula, every irreducible curve on $\widetilde{X}$ with negative self-intersection number is either a $(-1)$ - or a $(-2)$-curve. Moreover, one of the following holds (see [Bre80, HW81]):

(i) $K_{X}^{2}=9$ and $\tilde{X} \cong X \cong \mathbb{P}^{2}$;

(ii) $K_{X}^{2}=8$ and $\widetilde{X} \cong X \cong \mathbb{F}_{1}$;

(iii) $K_{X}^{2}=8$ and $\tilde{X} \cong X \cong \mathbb{P}^{1} \times \mathbb{P}^{1}$;

(iv) $K_{X}^{2}=8, \widetilde{X} \cong \mathbb{F}_{2}$, and $X$ is a quadric cone in $\mathbb{P}^{3}$;

(v) $K_{X}^{2} \leqslant 7$, and there exists an $\operatorname{Aut}^{0}(X)$-equivariant diagram

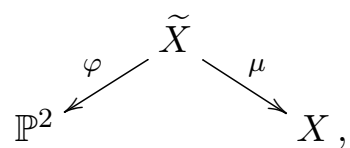

where $\varphi$ is a suitable contraction of $(-1)$-curves.

Moreover, it follows from the Kawamata-Viehweg vanishing and the exponential exact sequence that the group $\operatorname{Pic}(X)$ is torsion-free.

Corollary 2.2. Let $G$ be a connected algebraic subgroup in $\operatorname{Aut}(X)$. Suppose that $d=K_{X}^{2} \leqslant 7$. Then $G$ is isomorphic to a subgroup of the following group:

$$
\left\{\left(\begin{array}{ccc}
a_{11} & 0 & 0 \\
a_{21} & a_{22} & 0 \\
a_{31} & a_{32} & 1
\end{array}\right) \in \mathrm{GL}_{3}(\mathbb{k}) \mid a_{i j} \in \mathbb{k}, a_{11} \neq 0, a_{22} \neq 0\right\} \cong \mathbb{U}_{3} \rtimes \mathbb{G}_{\mathrm{m}}^{2} .
$$

In particular, the group $G$ is solvable. If $G$ is reductive and non-trivial, then $G \cong \mathbb{G}_{\mathrm{m}}$ or $G \cong \mathbb{G}_{\mathrm{m}}^{2}$. Similarly, if $G$ is unipotent, then $G \cong \mathbb{G}_{\mathrm{a}}$ or $G \cong \mathbb{G}_{\mathrm{a}}^{2}$ or $G \cong \mathbb{U}_{3}$.

Proof. This follows from the fact that the diagram (2.1) is $G$-equivariant.

Example 2.3 ([CT88, Proposition 8.1]). Suppose that $d=7$ and $X$ is singular. The surface $X$ is unique. The morphism $\varphi$ is the blow-up of two points, $\tilde{X}$ contains a unique $(-2)$-curve, and Type $(X)=\mathrm{A}_{1}$. The surface $X$ contains two $(-1)$-curves. The dual graph of the curves with negative self-intersection numbers has the form

where $\bullet$ denotes a $(-1)$-curve and $\circ$ denotes the (-2)-curve. Using (2.1), we see that

$$
\operatorname{Aut}^{0}(X) \cong \operatorname{Aut}\left(\mathbb{P}^{2}, \ell, P\right) \cong \mathbb{B}_{3},
$$

where $\ell$ is a line on $\mathbb{P}^{2}$ and $P$ is a point in $\ell$. Note that $X$ is the surface $48^{\circ}$.

The type Type $(X)$ does not always determine the dual graph of curves with negative selfintersection numbers in $\widetilde{X}$. However, this graph is always determined by the type Type $(X)$ and 


\section{Del Pezzo surfaces}

the number of $(-1)$-curves in $\widetilde{X}$. In the following, we denote by $\#(X)$ the number of $(-1)$-curves in the surface $\widetilde{X}$.

ExAmple 2.4. If $d=6$ and Type $(X)=\mathrm{A}_{1}$, then $X$ is one of the surfaces $45^{\circ}$ or $46^{\circ}$ in Table 3. If $X$ is the surface $45^{\circ}$, then $\#(X)=3$. On the other hand, if $X$ is the surface $46^{\circ}$, then $\#(X)=4$. The dual graph of curves with negative self-intersection numbers in $\widetilde{X}$ is given in Appendix A.

Using the Riemann-Roch formula and Kawamata-Viehweg vanishing, we get $\operatorname{dim}\left|-K_{X}\right|=d$. Let $\Phi: X \rightarrow \mathbb{P}^{d}$ be the rational map given by $\left|-K_{X}\right|$. The linear system $\left|-K_{X}\right|$ does not have fixed components, and it contains a smooth elliptic curve (see [Dem80]). Using this fact, one can prove the following.

TheOREm 2.5 ([Dem80, HW81]). The following assertions hold:

(i) If $d \geqslant 2$, then $\left|-K_{X}\right|$ is base point free, so that $\Phi$ is a morphism.

(ii) If $d \geqslant 3$, then $-K_{X}$ is very ample, so that $\Phi$ is an embedding.

(iii) If $d=3$, then $\Phi(X)$ is a cubic surface in $\mathbb{P}^{3}$.

(iv) If $d \geqslant 4$, then $\Phi(X)$ is an intersection of quadrics in $\mathbb{P}^{d}$.

(v) If $d=2$, then $\Phi$ is a double cover that is branched over a possibly reducible quartic curve, so that $X$ is a hypersurface in $\mathbb{P}(1,1,1,2)$ of degree 4 .

(vi) If $d=1$, then $\left|-K_{X}\right|$ is an elliptic pencil, its base locus consists of one point $O \notin \operatorname{Sing}(X)$, and every curve in $\left|-K_{X}\right|$ is irreducible and smooth at $O$.

(vii) If $d=1$, then $\left|-2 K_{X}\right|$ defines a double cover $X \rightarrow \mathbb{P}(1,1,2)$ branched over a sextic curve, so that $X$ is a hypersurface in $\mathbb{P}(1,1,2,3)$ of degree 6 .

The number of $(-1)$-curves in $\widetilde{X}$ is finite.

Definition 2.6. An irreducible curve $L \subset X$ is a line if $L=\mu(\widetilde{L})$ for a $(-1)$-curve $\widetilde{L} \subset \widetilde{X}$.

If $d \geqslant 3$, then lines in $X$ are usual (projective) lines in $\Phi(X) \subset \mathbb{P}^{d}$. Conversely, if $d \geqslant 3$, then lines in $\Phi(X)$ are lines in the sense of Definition 2.6. Moreover, if $d=2$, then lines in $X$ are smooth rational curves. Furthermore, if $d=1$ and $L$ is a line in $X$, then

- either $L$ is singular curve in $\left|-K_{X}\right| \operatorname{such}$ that $\operatorname{Sing}(L) \subset \operatorname{Sing}(X)$,

- or $L$ is a smooth rational curve that does not contain the base point of the pencil $\left|-K_{X}\right|$.

Note that $\#(X)$ is the number of lines in $X$. Then $\#(X)>0$ unless $X$ is $\mathbb{P}^{2}, \mathbb{P}^{1} \times \mathbb{P}^{1}$, or $\mathbb{P}(1,1,2)$.

Lemma 2.7. Assume that $d \geqslant 3$. Let $P$ be a point in $X$, and let $\#(X, P)$ be the number of lines in $X$ passing through $P$.

(i) If $P \in X$ is smooth, then

$$
\#(X, P) \leqslant \begin{cases}3 & \text { if } d=3 \\ 2 & \text { if } d \geqslant 4\end{cases}
$$

(ii) If $P \in X$ is singular, then

$$
\#(X, P) \leqslant \begin{cases}6 & \text { if } d=3 \\ 4 & \text { if } d=4, \\ 3 & \text { if } d \geqslant 5 .\end{cases}
$$




\section{Cheltsov and Yu. Prokhorov}

Proof. Let $L_{1}, \ldots, L_{r}$ be all the lines on $X$ passing through $P$, and let $\mathbb{T}_{P, X} \subset \mathbb{P}^{d}$ be the embedded tangents space to $X$ at the point $P$. Then

$$
\bigcup_{i=1}^{r} L_{i} \subseteq X \cap \mathbb{T}_{P, X} .
$$

If $P \in X$ is a smooth point, then $\operatorname{dim} \mathbb{T}_{P, X}=2$, so that $r \leqslant d$. Moreover, if $d \geqslant 4$, then $r \leqslant 2$ because $X$ is an intersection of quadrics in this case. Thus, we may assume that $P$ is a singular point of the surface $X$. Then $\operatorname{dim} \mathbb{T}_{P, X}=3$ since $P$ is a Du Val singular point of the surface $X$. Hence, if $d \geqslant 4$, then $r \leqslant 4$ because $X$ is an intersection of quadrics in this case.

Suppose that $d=3$. We may assume that $P=(0: 0: 0: 1)$. Then $X$ is given in $\mathbb{P}^{3}$ by

$$
x_{3} q_{2}\left(x_{0}, x_{1}, x_{2}\right)+q_{3}\left(x_{0}, x_{1}, x_{2}\right)=0,
$$

where $q_{2}$ and $q_{3}$ are homogeneous forms of degree 2 and 3 , respectively. Then the (set-theoretic) union of the lines $L_{1}, \ldots, L_{r}$ is given by the system of equations $q_{2}=q_{3}=0$, so that $r \leqslant 6$.

To complete the proof, we may assume that $d \geqslant 5$. We only consider the case $d=5$ since the proof is similar in the remaining cases. Let us show that $r \leqslant 3$. To do this, suppose that $r \geqslant 4$. Let us seek a contradiction.

Let $Q$ be a point in $X$ that is not contained in any line in $X$ (it exists since $\#(X)$ is finite). Keeping in mind that the Zariski tangent space of the surface $X$ at the point $P$ is threedimensional, we conclude that there exists a hyperplane $H$ in $\mathbb{P}^{5}$ that contains the lines $L_{1}, L_{2}$, $L_{3}, L_{4}$ and the point $Q$. Then

$$
\left.H\right|_{X}=C+\sum_{i=1}^{4} L_{i},
$$

where $C$ is a curve in $X$ that passes through $Q$. Counting degrees, we see that $\operatorname{deg}(C) \leqslant 1$, so that $C$ is a line, which contradicts the choice of the point $Q$.

Lemma 2.8. Suppose that $d \leqslant 7$. For any singular point of $X$, there is a line passing through it.

Proof. The required assertion follows from the existence of the diagram (2.1).

Since the Du Val singularities are $\mathbb{Q}$-factorial, $\rho(X)$ is equal to the rank of the Weil divisor class group $\mathrm{Cl}(X)$.

Lemma 2.9. Suppose that $d \leqslant 7$. Then the following assertions hold:

(i) The group $\mathrm{Cl}(X)$ is generated by the classes of lines in $X$.

(ii) Let $\mathrm{Cl}(X)_{\text {tors }} \subset \mathrm{Cl}(X)$ be the torsion subgroup, and let $n$ be the order of the group $\mathrm{Cl}(X)_{\text {tors }}$. There is a Galois abelian cover $\pi: Y \rightarrow X$ of degree $n$ which is étale outside of $\operatorname{Sing}(X)$, where $Y$ is a $D u$ Val del Pezzo surface such that $K_{Y}^{2}=d n$, so that $n \leqslant 9 / d$.

(iii) If $\rho(X)=1$ and $X$ contains two distinct lines $L$ and $L^{\prime}$, then $L \nsim L^{\prime}$ and $L \sim_{\mathbb{Q}} L^{\prime}$.

(iv) Every extremal ray of the Mori cone $\overline{\mathrm{NE}}(X)$ is generated by the class of a line.

(v) For every effective divisor $D \in \mathrm{Cl}(X)$, there are $a_{0}, a_{1}, \ldots, a_{r} \in \mathbb{Z}_{\geqslant 0}$ such that

$$
D \sim a_{0}\left(-K_{X}\right)+\sum_{i=1}^{r} a_{i} L_{i},
$$

where $L_{1}, \ldots, L_{r}$ are lines in $X, r=\#(X)$, and $a_{0}=0$ if $d \neq 1$. 


\section{Del Pezzo surfaces}

Proof. Assertion (ii) follows from a well-known construction; see, for example, [Rei87, § 3.6].

To prove assertion (iii), observe that $L \sim_{\mathbb{Q}} L^{\prime}$ because the numerical and $\mathbb{Q}$-linear equivalences on the surface $X$ coincide. But $L \not L^{\prime}$ because otherwise, $X$ would contain a pencil of lines, which contradicts $\#(X)<\infty$.

Assertion (iv) follows from Lemma 2.12 below.

Let us prove assertion (v). Let $\widetilde{D}$ be the proper transform on $\widetilde{X}$ of the divisor $D$. To prove assertion (v), it is sufficient to show that the divisor $\widetilde{D}$ is rationally equivalent to a convex integral linear combination of $(-1)$ and $(-2)$-curves (and $-K_{\widetilde{X}}$ if $d=1$ ).

We may assume that $\widetilde{D}$ is an irreducible curve.

Let us use induction on $\operatorname{dim}|\widetilde{D}|$. If $\operatorname{dim}|\widetilde{D}|=0$, then $\widetilde{D}$ is either a $(-1)$ - or a $(-2)$-curve by the Riemann-Roch formula and Kawamata-Viehweg vanishing. This is the base of induction.

Suppose that $\operatorname{dim}|\widetilde{D}| \geqslant 1$ and the required assertion holds for any effective divisor $\widetilde{D}^{\prime}$ on $\widetilde{X}$ such that $\operatorname{dim}\left|\widetilde{D}^{\prime}\right|<\operatorname{dim}|\widetilde{D}|$. Observe that $\widetilde{D}$ is nef. Thus, if $\widetilde{D}^{2}=0$, then $|\widetilde{D}|$ is base point free and gives a conic bundle $\widetilde{X} \rightarrow \mathbb{P}^{1}$, which must have at least one reducible fiber, because $\rho(\widetilde{X}) \geqslant 3$. Hence, if $\widetilde{D}^{2}=0$, then we can proceed by induction. Thus, we may assume that $\widetilde{D}^{2} \geqslant 1$.

If $\widetilde{D}$ is not ample, then $\widetilde{X}$ contains an irreducible curve $\widetilde{C}$ such that $\widetilde{D} \cdot \widetilde{C}=0$, which implies that $\widetilde{C}^{2}<0$ by the Hodge index theorem, so that $\widetilde{C}^{2}=-1$ or $\widetilde{C}^{2}=-2$, which gives

$$
\operatorname{dim}|\widetilde{D}-\widetilde{C}| \geqslant \operatorname{dim}|\widetilde{D}|-1 \geqslant 0 .
$$

Hence, if $\widetilde{D}$ is not ample, then there exists an effective divisor $\widetilde{D}^{\prime}$ such that $\widetilde{D} \sim \widetilde{D}^{\prime}+\widetilde{C}$, so that we can proceed by induction. Therefore, we may assume that $\widetilde{D}$ is ample.

Suppose that $\widetilde{D} \sim-K_{\widetilde{X}}$ and $K_{\widetilde{X}}^{2} \geqslant 2$. Then for any $(-1)$-curve $\widetilde{C}$ on $\widetilde{X}$, we have

$$
\operatorname{dim}|\widetilde{D}-\widetilde{C}| \geqslant \operatorname{dim}|\widetilde{D}|-2 \geqslant 0,
$$

so that there is an effective divisor $\widetilde{D}^{\prime}$ such that $\widetilde{D} \sim \widetilde{D}^{\prime}+\widetilde{C}$, and we can proceed by induction.

Finally, we assume that $\widetilde{D}$ is ample and $\widetilde{D} \nsim-K_{\widetilde{X}}$. There is an $a \in \mathbb{N}$ such that $\widetilde{D}+a K_{\widetilde{X}}$ is nef but not ample because the Mori cone of the surface $\tilde{X}$ is generated by $(-1)$-curves and (-2)-curves. Now using the Riemann-Roch formula and Kawamata-Viehweg vanishing, we see that the linear system $\left|\widetilde{D}+a K_{\widetilde{X}}\right|$ contains a divisor $\widetilde{D}^{\prime}$, so that

$$
\widetilde{D} \sim \widetilde{D}^{\prime}-a K_{\widetilde{X}}
$$

where $\widetilde{D}^{\prime}$ and $-K_{\tilde{X}}$ are both decomposable in the required form.

Corollary 2.10. One has \# $(X) \geqslant \rho(X)$. Moreover, if $\#(X)=\rho(X)$, then $\mathrm{Cl}(X)$ is torsion-free and every line in $X$ generates an extremal ray of the Mori cone $\overline{\mathrm{NE}}(X)$.

Corollary 2.11. Suppose that $d \leqslant 7$ and $X$ admits a faithful $\mathbb{G}_{\mathrm{a}}^{2}$-action. Then $\#(X)=\rho(X)$. Moreover, the complement of the open orbit coincides with the union of all the lines.

Proof. Let $U$ be the open $\mathbb{G}_{\mathrm{a}}^{2}$-orbit, let $\widetilde{U}=\mu^{-1}(U), \bar{U}=\varphi(\widetilde{U}), B=X \backslash U, \widetilde{B}=\widetilde{X} \backslash \widetilde{U}$, and $\bar{B}=\mathbb{P}^{2} \backslash \bar{U}$. Then

$$
U \cong \widetilde{U} \cong \bar{U} \cong \mathbb{A}^{2}
$$

so that the curve $\bar{B}$ must be a line. Then $\widetilde{B}$ has $\rho(\widetilde{X})$ components, and $B$ has $\rho(X)$ components. Since all $(-1)$-curves on $\widetilde{X}$ are contained in $\widetilde{B}$, we see that all the lines in $X$ are contained in $B$. This gives $\#(X) \leqslant \rho(X)$. But $\#(X) \geqslant \rho(X)$ by Corollary 2.10. 


\section{Cheltsov and Yu. Prokhorov}

Observe that a line $L$ on the surface $X$ generates an extremal ray of $\overline{\mathrm{NE}}(X)$ if and only if $L^{2} \leqslant 0$.

Lemma 2.12 ([Mor85, Proposition 1.2], [Pro01, §7.1]). Let $V$ be a surface that has Du Val singularities, and let $\psi: V \rightarrow Y$ be an extremal Mori contraction. Then

(i) either $\psi$ is a weighted blow-up of a smooth point in $Y$ with weights $(1, n)$, the exceptional curve $E$ is smooth and rational, one has $E^{2}=-1 / n$, and $E \cap \operatorname{Sing}(V)$ consists of one point which is of type $\mathrm{A}_{n-1}$ on $V$;

(ii) or $\psi$ is a conic bundle, one has $-K_{V} \cdot F=2$, and $F_{\text {red }} \cong \mathbb{P}^{1}$ for any its scheme fiber $F$, and if $F$ is not reduced, then one of the following three cases holds:

- $F \cap \operatorname{Sing}(V)$ consists of two singular points of type $\mathrm{A}_{1}$;

- $F \cap \operatorname{Sing}(V)$ consists of one singular point of type $\mathrm{A}_{3}$;

- $F \cap \operatorname{Sing}(V)$ consists of one singular point of type $\mathrm{D}_{n}$, where $n \geqslant 4$.

In case (i), we say that $\psi$ is a $(1, n)$-contraction.

Applying this lemma to our Du Val del Pezzo surface $X$, we get the following.

Corollary 2.13. Let $E$ be an irreducible curve on $X$ such that $E^{2}<0$. Then $E$ is a line on $X$ and $E$ is an exceptional divisor of a $(1, n)$-contraction for some $n \geqslant 1$.

Corollary 2.14. Suppose that there exists a birational morphism $\psi: X \rightarrow Y$ that is a $(1, n)$ contraction, and let $E$ be the exceptional curve of the morphism $\psi$. Then

- the point $\psi(E)$ is a smooth point of the surface $Y$;

- $Y$ is a $D u$ Val del Pezzo surface, $K_{Y}^{2}=d+n$, and $\rho(Y)=\rho(X)-1$;

- the point $\psi(E)$ is not contained in a line in $Y$.

Corollary 2.15. Let $\psi: X \rightarrow Y$ be a contraction of a proper face of the cone $\overline{\mathrm{NE}}(X)$. Then

- either the morphism $\psi$ is birational, $Y$ is a Du Val del Pezzo surface, and $\psi$ contracts a disjoint union of lines on the surface $X$;

- or the morphism $\psi$ is a conic bundle and $Y \cong \mathbb{P}^{1}$.

If a del Pezzo surface $X$ is smooth and $\rho(X) \geqslant 2$, then $X$ always admits a conic bundle contraction. However, this is not always the case if $X$ has Du Val singularities.

Lemma 2.16. Let $X$ be a Du Val del Pezzo surface of degree $d$ with $\rho(X) \geqslant 2$.

(i) Assume that $d=3$. Then there exists a conic bundle structure $\psi: X \rightarrow \mathbb{P}^{1}$ if and only if $X$ contains a line $L$ that is contained in $X \backslash \operatorname{Sing}(X)$.

(ii) Assume that $d=4$. Then there exists a conic bundle structure $\psi: X \rightarrow \mathbb{P}^{1}$ if and only if there is a double cover $\pi: X \rightarrow \mathbb{P}^{1} \times \mathbb{P}^{1}$ branched over a curve of degree $(2,2)$.

Proof. If $d=3$, then $X$ is a cubic surface in $\mathbb{P}^{3}$, so that every conic bundle $\psi: X \rightarrow \mathbb{P}^{1}$ is given by the linear projection from some line in $X$ that does not contain singular points of the surface $X$, so that $X$ admits a conic bundle contraction if and only if such a line exists.

Assume that $d=4$. If there is a double cover $X \rightarrow \mathbb{P}^{1} \times \mathbb{P}^{1}$ branched over a curve of degree $(2,2)$, then composing it with a projection to one of the factors, we obtain the required conic bundle. Thus, we may assume that there exists a conic bundle $\psi: X \rightarrow \mathbb{P}^{1}$. Let $C$ be its general fiber. Then $\left|-K_{X}-C\right|$ is base point free and gives another conic bundle $\psi^{\prime}: X \rightarrow \mathbb{P}^{1}$. Let $\pi=\psi \times \psi^{\prime}$. Then $\pi: X \rightarrow \mathbb{P}^{1} \times \mathbb{P}^{1}$ is the required double cover. 


\section{Del Pezzo surfaces}

\section{The Fano-Weil index of Du Val del Pezzo surfaces}

Recall from Section 1 that $\tau(X)$ is the Fano-Weil index of a del Pezzo surface $X$.

Lemma 3.1. Let $X$ be a Du Val del Pezzo surface with $\rho(X)=1$ and $K_{X}^{2} \geqslant 3$. Then $\tau(X)=K_{X}^{2}$.

Proof. Let $d:=K_{X}^{2}$ and $D:=K_{X}+d L$, where $L$ is a line on $X$. If $D \sim 0$, then we are done. Thus, we may assume that $D \nsim 0$. Since $D \sim_{\mathbb{Q}} 0$, the divisor $D$ is non-trivial torsion in $\operatorname{Cl}(X)$. Let $n$ be its order. Then

by Lemma 2.9 , so that either $d=3$ or $d=4$.

$$
2 \leqslant n \leqslant \frac{9}{d}
$$

Suppose that $d=4$. Then either Type $(X)=\mathrm{A}_{3} 2 \mathrm{~A}_{1}$ or Type $(X)=\mathrm{D}_{5}$ by [CT88, Proposition 6.1]. In the former case, we see that $4 L$ is a Cartier divisor, so that $D \sim 0 \operatorname{since} \operatorname{Pic}(X)$ is torsion-free. In the latter case, $L$ is the unique line in $X$ by [CT88, Proposition 6.1], so that $D \sim 0$ by Lemma 2.9. Thus, in both cases we obtain a contradiction with our assumption that $D \nsim 0$.

Thus, we see that $d=3$. Then either $n=2$ or $n=3$. If $n=2$, we have

$$
K_{X}+3(L+D) \sim 4 K_{X}+12 L \sim 4\left(K_{X}+3 D\right) \sim 0
$$

so that $\tau(X)=3$. If $n=3$, then $X \cong \mathbb{P}^{2} / \boldsymbol{\mu}_{3}$ by Lemma 2.9 , which implies that Type $(X)=3 \mathrm{~A}_{2}$. In this case, the divisor $3 L$ is Cartier, which gives $D \sim 0$ because $\operatorname{Pic}(X)$ is torsion-free.

The number $\tau(X)$ divides the degree $d$ of the del Pezzo surface $X$, so that $\tau(X)=1$ if $d=1$. If $d \geqslant 2$, then the Fano-Weil index $\tau(X)$ is closely related to the following notion.

Definition 3.2. A del Pezzo surface $X$ is said to be weakly minimal if $X$ does not contain lines that are contained in the smooth locus of the surface $X$.

Remark 3.3. If $X$ is a weakly minimal Du Val del Pezzo surface and $\psi: X \rightarrow Y$ is a birational contraction, then $Y$ is also a weakly minimal Du Val del Pezzo surface by Corollary 2.14.

Now, we prove the following result.

Proposition 3.4. Let $X$ be a Du Val del Pezzo surface, and let $d:=K_{X}^{2}$.

(i) If $\tau(X)=d$, then $d \leqslant 6$ and $X$ is a hypersurface in $\mathbb{P}(1,2,3, d)$ of degree 6 given by

$$
y_{3}^{2}+y_{2}^{3}+\lambda_{1} y_{1}^{4} y_{2}+\lambda_{2} y_{1}^{6}+y_{d} \phi\left(y_{1}, y_{2}, y_{d}\right)=0,
$$

where $\phi$ is a polynomial of degree $6-d$ and $\lambda_{1}$ and $\lambda_{2} \in \mathbb{k}$ are such that $4 \lambda_{1}^{3}+27 \lambda_{2}^{2} \neq 0$,

(ii) If $\tau(X)=d / 2$, then $X$ is a hypersurface in $\mathbb{P}(1,1,2, d / 2)$ of degree 4 given by

$$
y_{2}^{2}+y_{1} y_{1}^{\prime}\left(y_{1}-y_{1}^{\prime}\right)\left(y_{1}-\lambda y_{1}^{\prime}\right)+x_{3} \phi\left(y_{1}, y_{1}^{\prime}, x_{3}\right)=0,
$$

where $\phi$ is a polynomial of degree $4-d / 2$ and $\lambda \in \mathbb{k}$ is such that $\lambda \neq 0$ and $\lambda \neq 1$.

(iii) If $d=6$ and $\tau(X)=2$, then $X$ is a hypersurface in $\mathbb{P}(1,1,1,2)$ of degree 3 given by

$$
\psi\left(y_{1}, y_{1}^{\prime}, y_{1}^{\prime \prime}\right)+y_{2} \phi\left(y_{1}, y_{1}^{\prime}, y_{1}^{\prime \prime}\right)=0
$$

where $\psi$ and $\phi$ are polynomials of degree 3 and 1 , respectively.

Proof. Let us only prove assertion (i) since assertions (ii) and (iii) can be proved similarly. Let $C$ be a general curve in $\left|-K_{X}\right|$. Then $C$ is a smooth elliptic curve (see, for example, [Dem80]). 


\section{Cheltsov and Yu. Prokhorov}

Suppose that $\tau(X)=d$. Then $-K_{X} \sim d A$, where $A$ is a Weil divisor on $X$. Consider the natural homomorphism of graded algebras

$$
\Phi: \mathrm{R}(X, A):=\bigoplus_{n \geqslant 0} H^{0}\left(X, \mathscr{O}_{X}(n A)\right) \longrightarrow \bigoplus_{n \geqslant 0} H^{0}\left(C, \mathscr{O}_{C}(n A)\right)=: \mathrm{R}(C, A) .
$$

By the Kawamata-Viehweg vanishing, it is surjective. Note that $\mathscr{O}_{C}(A)$ is a line bundle of degree 1 . It is well known that $\mathrm{R}(C, A)$ is generated by three elements $\bar{y}_{1}, \bar{y}_{2}, \bar{y}_{3}$ with $\operatorname{deg} \bar{y}_{i}=i$ such that

$$
\bar{y}_{3}^{2}+\bar{y}_{2}^{3}+\lambda_{1} \bar{y}_{1}^{4} \bar{y}_{2}+\lambda_{2} \bar{y}_{1}^{6}=0
$$

for some $\lambda_{1}$ and $\lambda_{2}$ in $\mathbb{k}$ such that $4 \lambda_{1}^{3}+27 \lambda_{2}^{2} \neq 0$. The kernel of $\Phi$ is generated by a homogeneous element $y_{d}$ of degree $d$. Take arbitrary elements $y_{1}, y_{2}$, and $y_{3}$ in $\mathrm{R}(X, A)$ such that $\Phi\left(y_{i}\right)=\bar{y}_{i}$. Then $\mathrm{R}(X, A)$ is generated by $y_{1}, y_{2}, y_{3}$, and $y_{d}$. This gives us an embedding

$$
X \cong \operatorname{Proj}(\mathrm{R}(X, A)) \longleftrightarrow \operatorname{Proj}\left(\mathbb{k}\left[y_{1}, y_{2}, y_{3}, y_{d}\right]\right) \cong \mathbb{P}(1,2,3, d),
$$

whose image is given by an equation of the required form.

Remark 3.5. The embedding of the surface $X$ described in Proposition 3.4 is almost canonical. It only depends on the choice of the divisor class $A \in \mathrm{Cl}(X)$ such that $-K_{X} \sim \tau(X) A$, which is uniquely defined modulo $\tau(X)$-torsion. Thus, this embedding is $\operatorname{Aut}^{0}(X)$-equivariant.

Using Proposition 3.4, we can describe many del Pezzo surfaces.

Example 3.6. Suppose that $d=3, \tau(X)=3$, and Type $(X)=2 \mathrm{~A}_{2}$. Using Proposition 3.4, we see that $X$ is a hypersurface in $\mathbb{P}(1,2,3,3)$ of degree 3 that is given by

$$
y_{3} y_{3}^{\prime}=y_{2}\left(y_{2}-y_{1}^{2}\right)\left(y_{2}-\lambda y_{1}^{2}\right)
$$

where $\lambda \in \mathbb{k} \backslash\{0,1\}$.

Example 3.7. Suppose that $d=4, \tau(X)=2$, and Type $(X)=2 \mathrm{~A}_{1}$. Using Proposition 3.4, we see that $X$ is a hypersurface in $\mathbb{P}(1,1,2,2)$ of degree 4 that is given by

$$
y_{2} y_{2}^{\prime}=y_{1} y_{1}^{\prime}\left(y_{1}^{\prime}-y_{1}\right)\left(y_{1}^{\prime}-\lambda y_{1}\right) \text {, }
$$

where $\lambda \in \mathbb{k} \backslash\{0,1\}$. This surface is known as the Iskovskikh surface (see [KST85]).

Similarly, we can use Proposition 3.4 to prove the following result.

Theorem 3.8. Let $X$ be a $D u$ Val del Pezzo surface, and let $d:=K_{X}^{2}$. Suppose that $d \geqslant 3$ and $\tau(X)>1$ and that the surface $X$ is singular. Then $X$ is a hypersurface in a weighted projective space $\mathbb{P}$ corresponding to one of the possibilities in Table 1 below:

Proof. The required assertion follows from Proposition 3.4. Let us show this in the case $d=3$. Suppose that $d=3$ and $\tau(X)>1$. Observe that $\tau(X)$ must divide $d$. Thus, we have $\tau(X)=3$. By Proposition 3.4, the surface $X$ is a surface in $\mathbb{P}(1,2,3,3)$ given by

$$
y_{3}^{2}+y_{2}^{3}+\lambda_{1} y_{1}^{4} y_{2}+\lambda_{2} y_{1}^{6}+\lambda_{3} x_{3}^{2}+\lambda_{4} x_{3} y_{1} y_{2}+\lambda_{5} x_{3} y_{1}^{3}=0
$$

for some $\lambda_{1}, \lambda_{2}, \lambda_{3}, \lambda_{4}$, and $\lambda_{5}$ in $\mathbb{k}$. If $\lambda_{3} \neq 0$, then completing the square, we reduce this equation to the defining equation of one the surfaces $16^{\circ}, 21^{\circ}$, or $23^{\circ}$. Thus, we may assume that $\lambda_{3}=0$. 
Del Pezzo surfaces

TABLE 1.

\begin{tabular}{|c|c|c|c|c|c|c|}
\hline № & $d$ & $\rho$ & Type & $\tau$ & $\mathbb{P}$ & Equation of $X$ \\
\hline $23^{\circ}$ & \multirow{6}{*}{3} & 3 & $2 \mathrm{~A}_{2}$ & \multirow{6}{*}{3} & \multirow{6}{*}{$\mathbb{P}(1,2,3,3)$} & see Example 3.6 \\
\hline $21^{\circ}$ & & 2 & $2 \mathrm{~A}_{2} \mathrm{~A}_{1}$ & & & $y_{3} y_{3}^{\prime}=y_{2}^{2}\left(y_{2}+y_{1}^{2}\right)$ \\
\hline $16^{o}$ & & 1 & $3 \mathrm{~A}_{2}$ & & & $y_{3} y_{3}^{\prime}=y_{2}^{3}$ \\
\hline $18^{\circ}$ & & 2 & $\mathrm{~A}_{5}$ & & & $y_{3}^{2}=y_{2}^{3}+y_{1}^{6}+y_{1} y_{2} y_{3}^{\prime}$ \\
\hline $15^{\circ}$ & & 1 & $\mathrm{~A}_{5} \mathrm{~A}_{1}$ & & & $y_{3}^{2}=y_{2}^{3}+y_{3}^{\prime} y_{1} y_{2}$ \\
\hline $14^{\circ}$ & & 1 & $\mathrm{E}_{6}$ & & & $y_{3}^{2}=y_{2}^{3}+y_{3}^{\prime} y_{1}^{3}$ \\
\hline $28^{\circ}$ & \multirow{3}{*}{4} & 2 & $\mathrm{~A}_{3} \mathrm{~A}_{1}$ & \multirow{3}{*}{4} & \multirow{3}{*}{$\mathbb{P}(1,2,3,4)$} & $y_{3}^{2}=y_{1}^{6}+y_{2} y_{4}$ \\
\hline $25^{\circ}$ & & 1 & $\mathrm{~A}_{3} 2 \mathrm{~A}_{1}$ & & & $y_{3}^{2}=y_{2} y_{4}$ \\
\hline $24^{\circ}$ & & 1 & $\mathrm{D}_{5}$ & & & $y_{3}^{2}=y_{2}^{3}+y_{1}^{2} y_{4}$ \\
\hline $35^{\circ}$ & \multirow{6}{*}{4} & 4 & $2 \mathrm{~A}_{1}$ & \multirow{6}{*}{2} & \multirow{6}{*}{$\mathbb{P}(1,1,2,2)$} & see Example 3.7 \\
\hline $34^{\circ}$ & & 3 & $3 \mathrm{~A}_{1}$ & & & $y_{2} y_{2}^{\prime}=y_{1}^{2} y_{1}^{\prime}\left(y_{1}^{\prime}+y_{1}\right)$ \\
\hline $30^{\circ}$ & & 2 & $4 \mathrm{~A}_{1}$ & & & $y_{2} y_{2}^{\prime}=y_{1}^{2} y_{1}^{\prime 2}$ \\
\hline $29^{\circ}$ & & 2 & $\mathrm{~A}_{2} 2 \mathrm{~A}_{1}$ & & & $y_{2} y_{2}^{\prime}=y_{1}^{3} y_{1}^{\prime}$ \\
\hline $31^{\circ}$ & & 3 & $\mathrm{~A}_{3}$ & & & $y_{2}^{2}=y_{2}^{\prime} y_{1} y_{1}^{\prime}+y_{1}^{4}+y_{1}^{\prime 4}$ \\
\hline $26^{\circ}$ & & 2 & $\mathrm{D}_{4}$ & & & $y_{2}^{2}=y_{2}^{\prime} y_{1}^{2}+y_{1}^{\prime 4}$ \\
\hline $36^{\circ}$ & 5 & 1 & $\mathrm{~A}_{4}$ & 5 & $\mathbb{P}(1,2,3,5)$ & $y_{3}^{2}+y_{2}^{3}+y_{1} y_{5}=0$ \\
\hline $42^{\circ}$ & \multirow{4}{*}{6} & 1 & $\mathrm{~A}_{2} \mathrm{~A}_{1}$ & 6 & $\mathbb{P}(1,2,3)$ & - \\
\hline $45^{\circ}$ & & 3 & $\mathrm{~A}_{1}$ & \multirow{2}{*}{2} & \multirow{2}{*}{$\mathbb{P}(1,1,1,2)$} & $y_{1}^{\prime \prime} y_{2}=y_{1} y_{1}^{\prime}\left(y_{1}-y_{1}^{\prime}\right)$ \\
\hline $44^{\circ}$ & & 2 & $2 \mathrm{~A}_{1}$ & & & $y_{1}^{\prime \prime} y_{2}=y_{1}^{2} y_{1}^{\prime}$ \\
\hline $43^{\circ}$ & & 2 & $\mathrm{~A}_{2}$ & 3 & $\mathbb{P}(1,1,2,3)$ & $y_{1} y_{3}=y_{2}^{2}-y_{1}^{\prime 4}$ \\
\hline $50^{\circ}$ & 8 & 1 & $\mathrm{~A}_{1}$ & 4 & $\mathbb{P}(1,1,2)$ & - \\
\hline
\end{tabular}

If $\lambda_{4} \neq 0$, then we can use a coordinate change $x_{3} \mapsto \alpha x_{3}+\beta y_{1}^{3}$ and $y_{2} \mapsto \gamma y_{2}+\delta y_{1}^{2}$ for appropriate $\alpha, \beta, \gamma$, and $\delta$ in $\mathbb{k}$ to reduce our equation to

$$
y_{3}^{2}+y_{2}^{3}+\lambda_{2} y_{1}^{6}+x_{3} y_{1} y_{2}=0 .
$$

If $\lambda_{2}=0$, this equation defines the surface $15^{\circ}$. On the other hand, if $\lambda_{2} \neq 0$, we can scale the coordinates to get $\lambda_{2}=1$, so that we obtain the defining equation of the surface $18^{\circ}$.

We may assume that $\lambda_{3}=\lambda_{4}=0$. If $\lambda_{5}=0$, then $X$ has a non-Du Val singularity at $(0: 0: 0: 1)$, so that $\lambda_{5} \neq 0$. Then we reduce our equation to the defining equation of the surface $14^{\circ}$.

Remark 3.9. Using Theorem 3.8, we can easily obtain the anticanonical embedding of the surface $X \hookrightarrow \mathbb{P}^{d}$. For instance, if $d=3$ and $\tau(X)=3$, the map $\mathbb{P}(1,2,3,3) \rightarrow \mathbb{P}^{3}$ given by

$$
\left(y_{1}: y_{2}: y_{3}: y_{3}^{\prime}\right) \longmapsto\left(y_{1}^{3}: y_{1} y_{2}: y_{3}: y_{3}^{\prime}\right)
$$

defines an embedding $X \hookrightarrow \mathbb{P}^{3}$, so that $X$ is a cubic in $\mathbb{P}^{3}$ given by $23^{\circ} x_{0} x_{2} x_{3}=x_{1}\left(x_{1}-x_{0}\right)\left(x_{1}-\lambda x_{0}\right)$, where $\lambda \in \mathbb{k} \backslash\{0,1\} ;$ 


\section{Cheltsov and Yu. Prokhorov}

$21^{o} \quad x_{0} x_{2} x_{3}=x_{1}^{3}+x_{0} x_{1}^{2}$

$16^{\circ} \quad x_{0} x_{2} x_{3}=x_{1}^{3}$

$18^{\circ} \quad x_{0} x_{2}^{2}=x_{1}^{3}+x_{0}^{3}+x_{0} x_{3} x_{1}$

$15^{\circ} x_{0} x_{2}^{2}=x_{1}^{3}+x_{0} x_{3} x_{1}$;

$14^{o} \quad x_{0} x_{2}^{2}=x_{1}^{3}+x_{3} x_{0}^{2}$.

If $X$ is not weakly minimal, then $\tau(X)=1$. In particular, if the del Pezzo surface $X$ is smooth, then $\tau(X)=1$ unless $X \cong \mathbb{P}^{2}$ or $X \cong \mathbb{P}^{1} \times \mathbb{P}^{1}$. However, if $X$ is weakly minimal and $d \geqslant 2$, we cannot immediately conclude that $\tau(X)>1$. Let us present two examples.

Example 3.10. Let $X$ be a quintic del Pezzo surface with $\rho(X)=2$ admitting a conic bundle contraction $\psi_{1}: X \rightarrow \mathbb{P}^{1}$. It is easy to see from Lemma 2.12 that Type $(X)=\mathrm{A}_{3}$ and the second extremal contraction is a birational $(1,4)$-contraction $\psi_{2}: X \rightarrow \mathbb{P}^{2}$. Then $X$ is weakly minimal. We have an $\operatorname{Aut}(X)$-equivariant morphism $\psi=\left(\psi_{1}, \psi_{2}\right): X \rightarrow \mathbb{P}^{1} \times \mathbb{P}^{2}$ that is finite and birational onto its image, which is given by

$$
\phi\left(v_{0}, v_{1}, u_{0}, u_{1}, u_{2}\right)=0,
$$

where $\phi$ is a bihomogeneous polynomial such that its degree with respect to $v_{0}, v_{1}$ equals 1 , and its degree with respect to $u_{0}, u_{1}, u_{2}$ equals 2 since $\psi_{2}$ is birational, $\psi_{1}$ is a conic bundle, and $\rho(X)=2$. Let $P$ be the singular point of the surface $X$, and let $F$ be the fiber of $\psi_{1}$ that passes through $P$. We may assume that $\psi(P)=(1: 0 ; 0: 1: 0)$. Since $F$ is a multiple fiber of the conic bundle $\psi_{1}$, we may assume that $F$ is given by $u_{2}^{2}=0$. Then

$$
\phi=u_{2}^{2} v_{0}+q\left(u_{0}, u_{1}, u_{2}\right) v_{1},
$$

where $q$ is a quadratic form of rank 3 . Changing coordinates, we may assume that $q=u_{0}^{2}+u_{1} u_{2}$, so that $X$ is the surface $37^{\circ}$. Let $\tau=\tau(X)$, and let $A$ be a Weil divisor on $X$ such that $-K_{X} \sim \tau A$. Then $5=K_{X}^{2}=-\tau K_{X} \cdot A$ and $2=-K_{X} \cdot C=\tau A \cdot C$, where $C$ is a general fiber of the conic bundle $\psi_{1}$. Since $K_{X} \cdot A$ and $A \cdot C$ are integers, we have $\tau=1$.

Example 3.11. Let $X$ be a Du Val cubic surface in $\mathbb{P}^{3}$ with Type $(X)=\mathrm{A}_{4} \mathrm{~A}_{1}$. Then $\rho(X)=2$, and it follows from [BW79] that $X$ is unique up to isomorphism and can be given by the equation

$$
x_{0} x_{2} x_{3}+x_{0}^{2} x_{1}+x_{1}^{2} x_{3}=0
$$

so that $X$ is the surfaces $19^{\circ}$. Observe that $X$ contains exactly four lines

$$
L_{1}=\left\{x_{0}=x_{1}=0\right\}, \quad L_{2}=\left\{x_{1}=x_{3}=0\right\}, \quad L_{3}=\left\{x_{1}=x_{2}=0\right\}, \quad L_{4}=\left\{x_{0}=x_{3}=0\right\}
$$

and $-K_{X} \sim L_{1}+L_{2}+L_{3} \sim 2 L_{1}+L_{4} \sim 4 L_{1}-L_{2}$. Then $\operatorname{Cl}(X)=\mathbb{Z}\left[L_{1}\right] \oplus \mathbb{Z}\left[L_{2}\right]$ and $\tau(X)=1$.

Thus, the surfaces $19^{\circ}$ or $37^{\circ}$ are weakly minimal, and their Fano-Weil index is 1 . On the other hand, we have the following result.

Theorem 3.12. Let $X$ be a Du Val del Pezzo surface, and let $d:=K_{X}^{2}$. Suppose that $X$ is weakly minimal, $d \geqslant 3$, and $\tau(X)=1$. Then $X$ is one of the surfaces $19^{\circ}$ or $37^{\circ}$.

Proof. Observe that $\rho(X) \geqslant 2$ by Lemma 3.1. First, let us consider the case where $\rho(X)=2$. In this case, the Mori cone $\overline{\mathrm{NE}}(X)$ is generated by two lines $L_{1}$ and $L_{2}$ such that $L_{1} \cap L_{2} \neq \varnothing$. Without loss of generality, we may assume that $L_{1}^{2} \geqslant L_{2}^{2}$. Then

$$
-K_{X} \sim_{\mathbb{Q}} \alpha_{1} L_{1}+\alpha_{2} L_{2}
$$




\section{Del Pezzo surfaces}

for some $\alpha_{1} \in \mathbb{Q}_{>0}$ and $\alpha_{2} \in \mathbb{Q}_{>0}$. Since $-K_{X} \cdot L_{1}=-K_{X} \cdot L_{2}=1$ and $K_{X}^{2}=d$, we get

$$
\left\{\begin{array}{l}
\alpha_{1}+\alpha_{2}=d, \\
\alpha_{1} L_{1}^{2}+\alpha_{2} L_{1} \cdot L_{2}=1, \\
\alpha_{1} L_{1} \cdot L_{2}+\alpha_{2} L_{2}^{2}=1 .
\end{array}\right.
$$

Let $\psi_{1}: X \rightarrow Y_{1}$ and $\psi_{2}: X \rightarrow Y_{2}$ be the contractions of the extremal rays that are generated by the lines $L_{1}$ and $L_{2}$, respectively.

Assume that $\psi_{1}$ is a conic bundle. Since $X$ is weakly minimal, by Lemma 2.16(i) we have $d \geqslant 4$. In particular, we see that the anticanonical model of the surface $X$ is an intersection of quadrics. Let $C_{1}$ be a general fiber of $\psi_{1}$. Then $C_{1} \cdot L_{2}=1$ and $C_{1} \sim 2 L_{1}$. Hence, $L_{1} \cdot L_{2}=\frac{1}{2}$ and $L_{1}^{2}=0$. Then (3.13) gives $\alpha_{2}=2, \alpha_{1}=d-2$, and $L_{2}^{2}=1-d / 4$. To proceed, we may assume that $d \leqslant 6$. If $d=5$, then $L_{2}^{2}=-\frac{1}{4}$ and $\psi_{2}$ is a $(1,4)$-contraction by Lemma 2.12 , so that $X$ is the surface $37^{\circ}$. If $d=6$, then $\alpha_{1}=4, L_{2}^{2}=-\frac{1}{2}$, and $\psi_{2}$ is an $(1,2)$-contraction. Then $4 L_{1}+2 L_{2}$ is a Cartier divisor, so that

$$
-K_{X} \sim 4 L_{1}+2 L_{2}=2\left(2 L_{1}+L_{2}\right),
$$

which is impossible since $\tau(X)=1$. Finally, if $d=4$, then $L_{2}^{2}=0$ and $\psi_{2}$ is also a conic bundle. Since $2 L_{1}+2 L_{2}$ is Cartier, we have $-K_{X} \sim 2 L_{1}+2 L_{2}$ and so $\tau(X)>1$, which gives a contradiction.

Thus, we may assume that both $\psi_{1}$ and $\psi_{2}$ are birational.

Each line $L_{1}$ and $L_{2}$ contains exactly one singular point of the surface $X$ by Lemma 2.12. Let $P_{1}$ be the singular point contained in $L_{1}$, and let $P_{2}$ be the singular point contained in $L_{2}$. By Lemma 2.12, the points $P_{1}$ and $P_{2}$ are singular points of types $\mathrm{A}_{n_{1}}$ and $\mathrm{A}_{n_{2}}$ for some positive integers $n_{1}$ and $n_{2}$. Then

$$
-\frac{1}{n_{1}+1}=L_{1}^{2} \geqslant L_{2}^{2}=-\frac{1}{n_{2}+1}
$$

by Lemma 2.12 , so that $n_{1} \geqslant n_{2} \geqslant 1$.

Suppose that $P_{1} \neq P_{2}$. Then $L_{1} \cap L_{2}$ is a smooth point of the surface $X$, so that $L_{1} \cdot L_{2}=1$. Then (3.13) gives

$$
\left\{\begin{array}{l}
\alpha_{1}+\alpha_{2}=d \\
-\alpha_{1}+\alpha_{2}\left(n_{1}+1\right)=n_{1}+1, \\
\alpha_{1}\left(n_{2}+1\right)-\alpha_{2}=n_{2}+1 .
\end{array}\right.
$$

Also note that $d+n_{1}+n_{2} \leqslant 8$ since $\rho(\tilde{X})=10-d$. Eliminating $\alpha_{1}$ and $\alpha_{2}$, we get

$$
d\left(n_{1} n_{2}+n_{2}+n_{1}\right)=2 n_{1} n_{2}+3 n_{1}+3 n_{2}+4 .
$$

This give us the following solutions:

$-d=4, n_{1}=n_{2}=1, \alpha_{1}=\alpha_{2}=2,-K_{X} \sim_{\mathbb{Q}} 2\left(L_{1}+L_{2}\right)$,

$-d=3, n_{1}=n_{2}=2, \alpha_{1}=\alpha_{2}=3 / 2,-K_{X} \sim_{\mathbb{Q}} \frac{3}{2}\left(L_{1}+L_{2}\right)$,

$-d=3, n_{1}=4, n_{2}=1, \operatorname{Type}(X)=\mathrm{A}_{4} \mathrm{~A}_{1}, \alpha_{1}=\frac{5}{3}, \alpha_{2}=\frac{4}{3},-K_{X} \sim_{\mathbb{Q}} \frac{1}{3}\left(5 L_{1}+4 L_{2}\right)$.

If $d=4$, then $2\left(L_{1}+L_{2}\right)$ is a Cartier divisor, which gives $-K_{X} \sim 2\left(L_{1}+L_{2}\right)$, which is impossible. If $d=3$ and $n_{1}=n_{2}=2$, then $X$ is a cubic surface in $\mathbb{P}^{3}$, so that $X$ contains a line $L$ such that $L$ passes through $P_{1}$ and $P_{2}$, and $-K_{X} \sim L_{1}+L_{2}+L$, which gives $-K_{X} \sim_{\mathbb{Q}} 3 L$ because $-K_{X} \sim_{\mathbb{Q}} \frac{3}{2}\left(L_{1}+L_{2}\right)$. In this case, the divisor $3 L$ is Cartier, so that $-K_{X} \sim 3 L$, which contradicts 


\section{Cheltsov and Yu. Prokhorov}

$\tau(X)=1$. Thus, we conclude that $d=3, n_{1}=4$, and $n_{2}=1$. Then $X$ is the surface $19^{\circ}$. Hence, to proceed, we may assume that $P_{1}=P_{2}$.

Let $n=n_{1}=n_{2}$. Then $L_{1}^{2}=L_{2}^{2}=-1 /(n+1)$. Moreover, we have $L_{1} \cdot L_{2}=k /(n+1)$ for some $k \in \mathbb{Z}_{>0}$. Then (3.13) gives $\alpha_{1}=\alpha_{2}=d / 2$ and $2(n+1)=d(k-1)$, so that

$$
-K_{X} \sim_{\mathbb{Q}} \frac{1}{2} d\left(L_{1}+L_{2}\right) .
$$

Also note that $d+n \leqslant 8$ since $\rho(\tilde{X})=10-d$. Then $d \neq 5$ and $d \neq 7$ because $2(n+1)=$ $d(k-1)$. Likewise, if $d=6$, then $n=2$, so that $-K_{X} \sim_{\mathbb{Q}} 3\left(L_{1}+L_{2}\right)$ and $\mathrm{Cl}(X)$ is torsion-free by Lemma 2.9, which gives $-K_{X} \sim 3\left(L_{1}+L_{2}\right)$, which contradicts $\tau(X)=1$. Hence, either $d=3$, or $d=4$.

Suppose that $d=4$. Then $n=3$ because $2(n+1)=d(k-1)$ and $d+n \leqslant 8$. Since $\rho(X)=2$, we see that $X$ has a singular point of type $\mathrm{A}_{1}$. On the other hand, we have $-K_{X} \sim_{\mathbb{Q}} 2\left(L_{1}+L_{2}\right)$. Since $\tau(X)=1$, we have $-K_{X} \nsim 2\left(L_{1}+L_{2}\right)$, so that $K_{X}+2\left(L_{1}+L_{2}\right)$ is a non-trivial torsion element in $\mathrm{Cl}(X)$. Now, applying Lemma 2.9, we obtain a double cover $\pi: Y \rightarrow X$ that is étale outside of the point $L_{1} \cap L_{2}$. Then $Y$ is a del Pezzo surface of degree 8 that contains two singular points of type $\mathrm{A}_{1}$, which is absurd. This shows that $d \neq 4$.

Therefore, we see that $d=3$. Since $2(n+1)=d(k-1)$ and $d+n \leqslant 8$, we have $n=2$ or $n=5$. Since $X$ is a cubic surface in $\mathbb{P}^{3}$, it contains a line $L$ such that

$$
-K_{X} \sim L_{1}+L_{2}+L
$$

so that $L \sim_{\mathbb{Q}} \frac{1}{2}\left(L_{1}+L_{2}\right)$ because $-K_{X} \sim_{\mathbb{Q}} \frac{3}{2}\left(L_{1}+L_{2}\right)$. This gives $-K_{X} \sim_{\mathbb{Q}} 3 L$. But $-K_{X} \nsim 3 L$. In particular, we have $n \neq 2$ because $3 L$ is a Cartier divisor if $n=2$. We conclude that $n=5$. Now, using Lemma 2.9, we conclude that there is a finite Galois cover $\pi: Y \rightarrow X$ of degree $r \geqslant 2$ which is étale outside of the point $L_{1} \cap L_{2}$. Here, $r$ is the order of the torsion divisor $K_{X}+3 L$. By construction, the surface $Y$ is a del Pezzo surface of degree $r d$, so that either $r=2$, or $r=3$. If $r=3$, then $Y \cong \mathbb{P}^{2}$, which is impossible since $\rho(Y) \geqslant \rho(X)=2$. Thus, we have $r=2$. Then

$$
\begin{aligned}
-K_{X} & \sim 3 L-\left(K_{X}+3 L\right) \sim 3 L-\left(K_{X}+3 L\right)+4\left(K_{X}+3 L\right) \sim 3 L+3\left(K_{X}+3 L\right) \\
& \sim 3\left(K_{X}+4 L\right),
\end{aligned}
$$

which is impossible since $\tau(X)=1$. The obtained contradiction shows that $\rho(X) \neq 2$.

We see that $\rho(X) \geqslant 3$ and $X$ is singular. Then $d \leqslant 6$.

Let $\psi: X \rightarrow Y$ be an extremal Mori contraction. Since $\rho(X) \geqslant 3$, the morphism $\psi$ is birational, so that $\psi$ is a $(1, m)$-contraction of a line $L \subset X$ by Lemma 2.12. Then $Y$ is a weakly minimal Du Val del Pezzo surface such that $K_{Y}^{2}=d+m$ with $\rho(Y)=\rho(X)-1 \geqslant 2$ (see Remark 3.3). In particular, we have $d+m \neq 7$ because a Du Val del Pezzo surface of degree 7 is not weakly minimal (see Example 2.3). Note that $m \geqslant 2$ since $X$ is weakly minimal.

Consider the case $d=6$. Using the Noether formula, we see that $\rho(X)=3$ and Type $(X)=\mathrm{A}_{1}$. Then $m=2, K_{Y}^{2}=8$, and $\rho(Y)=2$, so that $Y \cong \mathbb{P}^{1} \times \mathbb{P}^{1}$. Then $K_{X} \sim \psi^{*} K_{Y}+2 L$ is divisible by 2 , which gives a contradiction. Thus, we have $d \neq 6$.

Consider the case $d=5$. Since $d+m \neq 7$, we have $m>2$. Then $\rho(X)=3$ and $\operatorname{Type}(X)=\mathrm{A}_{2}$ by the Noether formula, so that $X$ is not weakly minimal by [CT88, Proposition 8.5]. This contradicts our assumption.

Consider the case $d=4$. Then $m \neq 3$ since $d+m \neq 7$. If $m>3$, it follows from the Noether 


\section{Del Pezzo surfaces}

formula that $m=4, \rho(X)=3$, and Type $(X)=\mathrm{A}_{3}$, so that $Y \cong \mathbb{P}^{1} \times \mathbb{P}^{1}$, which gives

$$
-K_{X} \sim 2\left(\widetilde{F}_{1}+\widetilde{F}_{2}\right)
$$

where $\widetilde{F}_{1}$ and $\widetilde{F}_{2}$ are proper transforms on $X$ of the curves in $Y$ of bi-degree $(1,0)$ and $(0,1)$, respectively, that contain $\psi(L)$. This contradicts our assumption $\tau(X)=1$. So, we see that all extremal contractions on $X$ are birational $(1,2)$-contractions. Then $\tau(Y)>1$ since we already dealt with sextic del Pezzo surfaces. By Theorem 3.8, we see that $Y$ is one of the surfaces $45^{\circ}$, $44^{\circ}, 43^{\circ}$. If $Y$ is the surface $43^{\circ}$, then it has a birational $(1,3)$-contraction, so that $X$ also has a birational $(1,3)$-contraction by Corollary 2.14, which gives a contradiction. Then $Y$ is one of the surfaces $45^{\circ}$ or $44^{\circ}$. By Lemma 2.9(i), we have

$$
-K_{Y} \sim 2 \sum_{i=1}^{s} a_{i} M_{i}
$$

for some lines $M_{1}, \ldots, M_{s}$ on the surface $Y$ and some integers $a_{1}, \ldots, a_{s}$. Since $\psi(L)$ is a smooth point of $Y$ that does not lie on a line by Corollary 2.14, we obtain

$$
-K_{X} \sim 2 \sum_{i=1}^{s} a_{i} \widetilde{M}_{i}-2 L \sim 2\left(\sum_{i=1}^{s} a_{i} \widetilde{M}_{i}-L\right),
$$

where $\widetilde{M}_{i}$ is a proper transform on $X$ of the line $M_{i}$. This contradicts our assumption $\tau(X)=1$.

Finally, we consider the case $d=3$. Then $m \neq 4$ since $d+m \neq 7$. Moreover, since $X$ is weakly minimal, there exist no dominant morphisms from $X$ to a curve by Lemma 2.16(i), and the same holds for $Y$. Using this, we conclude that $m \neq 5$. Thus, we have the following possibilities:

- either $K_{Y}^{2}=5$ and $m=2$,

- or $K_{Y}^{2}=6$ and $m=3$.

Moreover, if $K_{Y}^{2}=5$, then $Y$ is not the surface $37^{\circ}$ because del Pezzo surface $37^{\circ}$ admits a dominant morphism to $\mathbb{P}^{1}$ (see Example 3.10). Therefore, we conclude that $\tau(Y)>1$ because we already dealt with weakly minimal Du Val del Pezzo surfaces of degrees 5 and 6 .

Now, using Theorem 3.8, we see that $K_{Y}^{2} \neq 5$ because $\rho(Y)>1$. Therefore, we have $K_{Y}^{2}=6$. Then $Y$ is the surface $43^{\circ}, 44^{\circ}$, or $45^{\circ}$, again by Theorem 3.8. If $Y$ is the surface $44^{\circ}$, then

$$
\frac{y_{1}}{y_{1}^{\prime \prime}}=\frac{y_{1}^{\prime 2}}{y_{2}}
$$

on the surface $Y$, so that the map $Y \rightarrow \mathbb{P}^{1}$ given by

$$
\left(y_{1}: y_{1}^{\prime}: y_{1}^{\prime \prime}: y_{2}\right) \longmapsto\left(y_{1}: y_{1}^{\prime \prime}\right)=\left(y_{1}^{\prime 2}: y_{2}\right)
$$

is a morphism, which gives a contradiction. Similarly, we obtain a contradiction when $Y$ is the surface $45^{\circ}$ because the map $Y \rightarrow \mathbb{P}^{1}$ given by

$$
\left(y_{1}: y_{1}^{\prime}: y_{1}^{\prime \prime}: y_{2}\right) \longmapsto\left(y_{1}: y_{1}^{\prime}\right)=\left(y_{1}^{\prime \prime}\left(y_{1}^{\prime}+y_{1}^{\prime \prime}\right): y_{2}\right)
$$

is a morphism in this case. Thus, we see that $Y$ is the surface $43^{\circ}$. Then $X$ is a cubic surface such that Type $(X)=2 \mathrm{~A}_{2}$. Now, using [BW79], we conclude that $X$ is one of the surfaces $23^{\circ}$, so that $\tau(X)=3$ by Theorem 3.8, which contradicts our assumption. 


\section{Cheltsov and Yu. Prokhorov}

\section{The proof of Theorem 1.1: Higher-degree cases}

Let $X$ be a Du Val del Pezzo surface of degree $d$ whose automorphism group $\operatorname{Aut}(X)$ is infinite. If $d \geqslant 8$, then $X$ is $\mathbb{P}^{2}, \mathbb{P}^{1} \times \mathbb{P}^{1}, \mathbb{F}_{1}$, or $\mathbb{P}(1,1,2)$. In each of these cases, the corresponding automorphism group is well known and listed in Table 3 (cases $53^{\circ}, 52^{\circ}, 51^{\circ}, 50^{\circ}$, respectively).

If $d \leqslant 7$, we have an $\operatorname{Aut}^{0}(X)$-equivariant diagram (2.1). If $d=7$, then $X$ is one of the del Pezzzo surfaces $48^{\circ}$ and $49^{\circ}$, and the morphism $\varphi$ in (2.1) is a blow-up of two (possibly infinitely near) points. From this we obtain the following.

Lemma 4.1. Let $X$ be a Du Val del Pezzo surface of degree 7, and let $U$ be the complement in the surface $X$ of the union of all lines. Then Theorem 1.1 holds for $X$, the subset $U$ is the open orbit of the group $\operatorname{Aut}^{0}(X)$, and $U \cong \mathbb{A}^{2}$.

All del Pezzo surfaces of degree 6 have infinite automorphism groups, so that all of them appear in our Table 3. These are the del Pezzo surfaces $42^{\circ}, 43^{\circ}, 44^{\circ}, 45^{\circ}, 46^{\circ}$, and $47^{\circ}$. Going through these six cases one by one, we obtain the following.

Lemma 4.2. Let $X$ be a Du Val del Pezzo surface of degree 6, and let $U$ be the complement in the surface $X$ of the union of all lines. Then Theorem 1.1 holds for $X$, the subset $U$ is the open orbit of the group $\operatorname{Aut}^{0}(X)$, and $U \cong \mathbb{A}^{2}$ in the cases $42^{\circ}, 43^{\circ}, 44^{\circ}$, and $45^{\circ}$.

Proof. If $X$ is the surface $47^{\circ}$, then $\varphi$ in (2.1) is a blow-up of three distinct non-collinear points, so that $X$ is toric and $\operatorname{Aut}^{0}(X) \cong \mathbb{G}_{\mathrm{m}}^{2}$. Likewise, if $X$ is the surface $45^{\circ}$, then $\varphi$ is the blow-up of three distinct collinear points in $\mathbb{P}^{2}$. Using this, it is not hard to see that $\operatorname{Aut}^{0}(X) \cong \mathbb{G}_{\mathrm{a}}^{2} \rtimes \mathbb{G}_{\mathrm{m}}$ in this case.

For infinitely near points, we use the notation of [Dol12]. If $X$ is the surface $42^{\circ}$, then the morphism $\varphi$ is the blow-up of three infinitely near collinear points $P_{1} \prec P_{2} \prec P_{3}$ in the plane $\mathbb{P}^{2}$ in the notation of [Dol12], which implies that $\operatorname{Aut}^{0}(X) \cong \mathbb{B}_{3}$. Similarly, if $X$ is the surface $43^{\circ}$, then $\varphi$ is the blow-up of three infinitely near non-collinear points $P_{1} \prec P_{2} \prec P_{3}$, which implies that $\operatorname{Aut}^{0}(X) \cong \mathbb{U}_{3} \rtimes \mathbb{G}_{\mathrm{m}}$. If $X$ is the surface $44^{\circ}$, then $\varphi$ is the blow-up of three collinear points $P_{1}, P_{2}$ and $P_{3}$ such that the points $P_{1}$ and $P_{2}$ are distinct and $P_{3} \succ P_{1}$, which implies that $\operatorname{Aut}^{0}(X) \cong \mathbb{B}_{2} \times \mathbb{B}_{2}$. Finally, if $X$ is the surface $46^{\circ}$, then $\varphi$ is the blow-up of three non-collinear points $P_{1}, P_{2}$, and $P_{3}$ such that $P_{1}$ and $P_{2}$ are distinct but $P_{3} \succ P_{1}$. Hence, in this case, we have $\operatorname{Aut}^{0}(X) \cong \mathbb{B}_{2} \times \mathbb{G}_{\mathrm{m}}$.

The last assertions follow from Corollary 2.11 in the cases $42^{\circ}, 43^{\circ}, 44^{\circ}$, and $45^{\circ}$, and it follows from Lemma 4.1 in the cases $46^{\circ}$ and $47^{\circ}$.

Similarly, all singular del Pezzo surfaces of degree 5 also have infinite automorphism groups. These are the surfaces $36^{\circ}, 37^{\circ}, 38^{\circ}, 39^{\circ}, 40^{\circ}$, and $41^{\circ}$ in Table 3 .

Lemma 4.3. Let $X$ be a Du Val del Pezzo surface of degree 5, and let $U$ be the complement in the surface $X$ of the union of all lines. Then Theorem 1.1 holds for $X$, the subset $U$ is the open orbit of the group $\operatorname{Aut}^{0}(X)$ in the cases $36^{\circ}, 37^{\circ}, 38^{\circ}, 39^{\circ}, 40^{\circ}$, and $U \cong \mathbb{A}^{2}$ in the cases $36^{\circ}$ and $37^{\circ}$.

Proof. If $X$ is weakly minimal and $\tau(X)>1$, then $X$ is the surface $36^{\circ}$ by Theorems 3.12 and 3.8. Then the group $\operatorname{Aut}^{0}(X)$ consists of the transformations that send the point $\left(y_{1}: y_{2}: y_{3}: y_{5}\right)$ to

$$
\begin{array}{r}
\left(y_{1}: t^{2} y_{2}+a y_{1}^{2}: t^{3} y_{3}+b y_{1}^{3}+c y_{1} y_{2}: t^{6} y_{5}-\left(a^{3}+b^{2}\right) y_{1}^{5}-\left(3 a^{2} t^{2}+2 b c\right) y_{1}^{3} y_{2}\right. \\
\left.-2 b t^{3} y_{1}^{2} y_{3}-\left(3 a t^{4}+c^{2}\right) y_{1} y_{2}^{2}-2 c t^{3} y_{2} y_{3}\right)
\end{array}
$$




\section{Del Pezzo surfaces}

where $t \in \mathbb{k}^{*}$ and $a, b, c \in \mathbb{k}$. This gives $\operatorname{Aut}^{0}(X) \cong \mathbb{U}^{3} \rtimes \mathbb{G}_{\mathrm{m}}$ (cf. Corollary 2.2; see Corollary B.8).

If $X$ is weakly minimal and $\tau(X)=1$, then $X$ is the del Pezzo surface $37^{\circ}$ by Theorem 3.12 , and its $\operatorname{Aut}^{0}(X)$-equivariant embedding $X \hookrightarrow \mathbb{P}^{1} \times \mathbb{P}^{2}$ is described in Example 3.10. In this case, the group $\operatorname{Aut}(X)$ contains a two-dimensional unipotent subgroup

$$
\left(v_{0}: v_{1} ; u_{0}: u_{1}: u_{2}\right) \longmapsto\left(v_{0}-\left(a_{1}^{2}+a_{2}\right) v_{1}: v_{1} ; u_{0}+a_{1} u_{2}: u_{1}-2 a_{1} u_{0}+a_{2} u_{2}: u_{2}\right)
$$

and a one-dimensional torus

$$
\left(v_{0}: v_{1} ; u_{0}: u_{1}: u_{2}\right) \longmapsto\left(v_{0}: t^{-2} v_{1} ; t u_{0}: t^{2} u_{1}: u_{2}\right),
$$

where $a_{1}, a_{2} \in \mathbb{k}$ and $t \in \mathbb{k}^{*}$. This implies that $\operatorname{Aut}(X) \cong \mathbb{G}_{\mathrm{a}}^{2} \rtimes \mathbb{G}_{\mathrm{m}}$, as required.

We may now assume that $X$ is not weakly minimal. Then there is a birational morphism $\psi: X \rightarrow Y$ such that $Y$ is one of the surfaces $42^{\circ}, 43^{\circ}, 44^{\circ}, 45^{\circ}$, and $\psi$ is a blow-up of a smooth point $P \in Y$. By Corollary 2.14 and Lemma 4.2, the point $P$ is contained in the open orbit of the group $\operatorname{Aut}^{0}(Y)$. Since $\operatorname{Aut}^{0}(X)$ is the connected component of the stabilizer in $\operatorname{Aut}^{0}(Y)$ of the point $P$, this gives the required description of the group $\operatorname{Aut}^{0}(X)$ in Table 3.

The last assertions follow from Corollary 2.11 in the cases $36^{\circ}$ and $37^{\circ}$, and it follows from Lemma 4.2 in the cases $38^{\circ}, 39^{\circ}$, and $40^{\circ}$.

Let us conclude this section by proving Theorem 1.1 for Du Val del Pezzo surfaces of degree 4.

Proposition 4.4. Theorem 1.1 holds for Du Val del Pezzo surfaces of degree 4.

Proof. Let $X$ be a Du Val del Pezzo surface of degree 4 such that the group $\operatorname{Aut}(X)$ is infinite. Suppose that $X$ is not weakly minimal. Then there exists a birational morphism $\psi: X \rightarrow Y$ such that $Y$ is a singular quintic Du Val del Pezzo surface, and $\psi$ is a blow-up of a smooth point $P \in Y$, which is not contained in a line by Corollary 2.14. Observe that the group $\operatorname{Aut}^{0}(X)$ is the connected component of the stabilizer in $\operatorname{Aut}^{0}(Y)$ of the point $P$. Using Lemma 4.3, we see that $Y$ must be one of the surfaces $36^{\circ}, 37^{\circ}, 38^{\circ}$ and that $P$ must be contained in the open orbit of the group $\operatorname{Aut}^{0}(Y)$. This implies that $X$ is one of the surfaces $27^{\circ}, 32^{\circ}, 33^{\circ}$, respectively. Now, it is not hard to check that $\operatorname{Aut}^{0}(X) \cong \mathbb{G}_{\mathrm{m}}$ in the cases $32^{\circ}$, $33^{\circ}$ and that $\operatorname{Aut}^{0}(X) \cong \mathbb{B}_{2}$ in the case $27^{\circ}$.

We may now assume that the surface $X$ is weakly minimal. Then $\tau(X)>1$ by Theorem 3.12. Using Theorem 3.8, we see that $X$ is one of the surfaces $24^{\circ}, 25^{\circ}, 26^{\circ}, 28^{\circ}, 29^{\circ}, 30^{\circ}, 31^{\circ}, 34^{\circ}$, $35^{\circ}$. Let us show that $\operatorname{Aut}(X)$ is infinite and that $\operatorname{Aut}^{0}(X)$ is described in Table 3.

Let $X$ be the surface $24^{\circ}$. Then $X$ is embedded into $\mathbb{P}(1,2,3,4)$ as a hypersurface that is given by the equation $y_{3}^{2}=y_{2}^{3}+y_{1}^{2} y_{4}$. This embedding is $\operatorname{Aut}^{0}(X)$-equivariant by Remark 3.5. Observe that $\operatorname{Aut}^{0}(X)$ contains the transformations

$$
\left(y_{1}: y_{2}: y_{3}: y_{4}\right) \longmapsto\left(y_{1}: y_{2}+a y_{1}^{2}: y_{3}+b y_{1}^{3}: y_{4}-\left(a^{3}-b^{2}\right) y_{1}^{4}-3 a^{2} y_{1}^{2} y_{2}+2 b y_{1} y_{3}-3 a y_{2}^{2}\right) \text {, }
$$

where $a \in \mathbb{k}$ and $b \in \mathbb{k}$. These transformations generate a subgroup in $\operatorname{Aut}^{0}(X)$ isomorphic to $\mathbb{G}_{\mathrm{a}}^{2}$. Moreover, the surface $X$ also admits an action of a one-dimensional torus which acts diagonally:

$$
\left(y_{1}: y_{2}: y_{3}: y_{4}\right) \longmapsto\left(t^{2} y_{1}: t^{2} y_{2}: t^{3} y_{3}: t^{2} y_{4}\right)
$$

where $t \in \mathbb{G}_{\mathrm{m}}$. The described transformations generate a subgroup that is isomorphic to $\mathbb{G}_{\mathrm{a}}^{2} \rtimes \mathbb{G}_{\mathrm{m}}$. Since $X$ has a singularity of type $\mathrm{D}_{5}$, it is not toric, so that $\operatorname{Aut}^{0}(X) \cong \mathbb{G}_{\mathrm{a}}^{2} \rtimes \mathbb{G}_{\mathrm{m}}$ (cf. Corollary B.8). 


\section{Cheltsov and Yu. Prokhorov}

Now we suppose that $X$ is the surface $25^{\circ}$. Then $\operatorname{Aut}(X)$ contains the transformations

$$
\gamma(a):\left(y_{1}: y_{2}: y_{3}: y_{4}\right) \longmapsto\left(y_{1}: y_{2}: y_{2}+a y_{1} y_{2}: y_{4}+2 a y_{1} x_{2}+a^{2} y_{1}^{2} y_{2}\right)
$$

for every $a \in \mathbb{k}$. These transformations generate a proper subgroup in $\operatorname{Aut}^{0}(X)$ isomorphic to $\mathbb{G}_{\mathrm{a}}$. Moreover, the surface $X$ also contains the transformations

$$
\delta\left(t_{1}, t_{2}\right):\left(y_{1}: y_{2}: y_{3}: y_{4}\right) \longmapsto\left(y_{1}: t_{1} t_{2}^{2} y_{2}: t_{1} t_{2} y_{3}: t_{1} y_{4}\right)
$$

for every $t_{1} \in \mathbb{k}^{*}$ and $t_{2} \in \mathbb{k}^{*}$. They generate a subgroup isomorphic to $\mathbb{G}_{\mathrm{m}}^{2}$. Observe that

$$
\gamma(a) \circ \delta\left(t_{1}, t_{2}\right)=\delta\left(t_{1}, t_{2}\right) \circ \gamma_{a t_{2}} .
$$

Therefore, all described transformations generate a subgroup in $\operatorname{Aut}^{0}(X)$ isomorphic to $\mathbb{B}_{2} \times \mathbb{G}_{\mathrm{m}}$. Then $\operatorname{Aut}^{0}(X) \cong \mathbb{B}_{2} \times \mathbb{G}_{\mathrm{m}}$ because $X$ does not admit an effective $\mathbb{G}_{\mathrm{a}}^{2}$-action by Corollary 2.11.

Now we suppose that $X$ is $28^{\circ}$. Then the group $\operatorname{Aut}(X)$ contains the transformations

$$
\left(y_{1}: y_{2}: y_{3}: y_{4}\right) \longmapsto\left(t y_{1}: t^{3} y_{2}: t^{3} y_{3}+a t^{3} y_{1} y_{2}: t^{3} y_{4}+2 a t^{3} y_{1} y 3+a^{2} t^{3} y_{1}^{2} y_{2}\right)
$$

for any $a \in \mathbb{k}$ and $t \in \mathbb{k}^{*}$. These transformations generate a subgroup in $\operatorname{Aut}^{0}(X)$ isomorphic to $\mathbb{B}_{2}$. Since all three lines on $X$ pass through one point, $X$ is not toric. Hence, $\operatorname{rk} \operatorname{Aut}^{0}(X)=1$. The surface $X$ does not admit an effective $\mathbb{G}^{2}$-action by Corollary 2.11 , so that $\operatorname{dim} \operatorname{Aut}^{0}(X)=2$, which implies $\operatorname{Aut}^{0}(X) \cong \mathbb{B}_{2}$.

Let $X$ be one of the surfaces $29^{\circ}$ or $30^{\circ}$. Then $\#(X)=4$. Let $L_{1}, L_{2}, L_{3}, L_{4}$ be the lines in $X$. Recall that $X$ is an intersection of two quadrics in $\mathbb{P}^{4}$. We have

$$
L_{1}+L_{2}+L_{3}+L_{4} \sim-K_{X},
$$

so that $L_{1}+L_{2}+L_{3}+L_{4}$ is cut out by a hyperplane $H \subset \mathbb{P}^{4}$. On the other hand, this curve forms a combinatorial cycle. Thus, if $X$ admits an effective $\mathbb{G}_{\mathrm{a}}$-action, then this action is trivial on each line among $L_{1}, L_{2}, L_{3}$, and $L_{4}$, so that it is trivial on $H$, which implies that the closure of any one-dimensional $\mathbb{G}_{\mathrm{a}}$-orbit is a line. The latter is impossible since $X$ contains finitely many lines. Therefore, we conclude that the surface $X$ does not admit an effective action of the group $\mathbb{G}_{\mathrm{a}}$. On the other hand, the equations of $X$ are binomial. This implies that $X$ admits a diagonal action of a two-dimensional torus. Hence, $\operatorname{Aut}^{0}(X) \cong \mathbb{G}_{\mathrm{m}}^{2}$.

Let $X$ be one of the surfaces $34^{\circ}$ or $35^{\circ}$. Then $X$ contains two lines $L_{1}$ and $L_{2}$ such that the intersection $L_{1} \cap L_{2}$ is a smooth point of $X$, and there exist an $\operatorname{Aut}^{0}(X)$-equivariant diagram

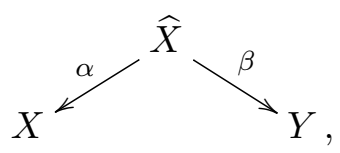

where $\alpha$ is a blow-up of the point $L_{1} \cap L_{2}$ and $\beta$ is the birational contraction of the proper transforms of the lines $L_{1}$ and $L_{2}$. Then we have the following possibilities:

- if $X$ is the surface $34^{\circ}$, then $Y$ is a cubic surface such that Type $(Y)=\mathrm{A}_{4} \mathrm{~A}_{1}$;

- if $X$ is the surface $35^{\circ}$, then $Y$ is a cubic surface such that Type $(Y)=2 \mathrm{~A}_{2}$.

Hence, it follows from Corollary 7.4 that $Y$ does not admit an effective action of the group $\mathbb{G}_{\mathrm{a}}$. Since $X$ contains three lines passing through one point, it is not toric. One the other hand, it is easy to see that $X$ admits an effective diagonal action of a one-dimensional torus.

To complete the proof, we may assume that $X$ is one of the surfaces $26^{\circ}$ or $31^{\circ}$. By Lemma 2.16(ii), there is a double cover $\pi: X \rightarrow \mathbb{P}^{1} \times \mathbb{P}^{1}$ branched over a curve $B$ of degree $(2,2)$. 


\section{Del Pezzo surfaces}

By construction, this double cover is $\operatorname{Aut}^{0}(X)$-equivariant, and the curve $B$ is $\operatorname{Aut}^{0}(X)$-invariant. Therefore, there exists an exact sequence of groups

$$
1 \longrightarrow \boldsymbol{\mu}_{2} \longrightarrow \operatorname{Aut}(X) \longrightarrow \operatorname{Aut}\left(\mathbb{P}^{1} \times \mathbb{P}^{1}, B\right) \text {. }
$$

If $X$ is the surface $26^{\circ}$, then $B$ is a union of irreducible smooth curves of degrees $(1,1),(1,0),(0,1)$, which intersect in one point, which implies that

$$
\operatorname{Aut}\left(\mathbb{P}^{1} \times \mathbb{P}^{1}, B\right) \cong \mathbb{B}_{2} \rtimes \boldsymbol{\mu}_{2} .
$$

This can be shown by taking the linear projection $\mathbb{P}^{1} \times \mathbb{P}^{1} \rightarrow \mathbb{P}^{2}$ from the singular point $\operatorname{Sing}(B)$, where we consider $\mathbb{P}^{1} \times \mathbb{P}^{1}$ as a quadric in $\mathbb{P}^{3}$. Thus, in this case, we have $\operatorname{Aut}^{0}(X) \cong \mathbb{G}_{\mathrm{a}} \rtimes_{(2)} \mathbb{G}_{\mathrm{m}}$ since $\operatorname{Aut}(X)$ contains a subgroup isomorphic to $\mathbb{G}_{\mathrm{a}} \rtimes_{(2)} \mathbb{G}_{\mathrm{m}}$ generated by transformations

$$
\left(y_{1}: y_{1}^{\prime}: y_{2}: y_{2}^{\prime}\right) \longmapsto\left(y_{1}: t y_{1}^{\prime}: t^{2} y_{2}+a t^{2} y_{1}^{2}: t^{4} y_{2}^{\prime}+2 a t^{4} y_{2}+a^{2} t^{4} y_{1}^{2}\right)
$$

where $a \in \mathbb{k}$ and $t \in \mathbb{k}^{*}$. Similarly, if $X$ is the surface $31^{\circ}$, then

$$
\text { Aut }\left(\mathbb{P}^{1} \times \mathbb{P}^{1}, B\right) \cong \mathbb{G}_{\mathrm{a}} \rtimes \boldsymbol{\mu}_{2}
$$

because $B$ is a union of two irreducible smooth curves of degree $(1,1)$, which intersect in one point. In this case, we have $\operatorname{Aut}^{0}(X) \cong \mathbb{G}_{\mathrm{a}}$ since the $\mathbb{G}_{\mathrm{a}}$-action lifts from $\mathbb{P}^{1} \times \mathbb{P}^{1}$ to the surface $X$.

Corollary 4.5. Theorem 1.1 holds for del Pezzo surfaces of degree at least 4.

\section{Del Pezzo surfaces of degree 1}

In this section, we prove Theorem 1.1 for del Pezzo surfaces of degree 1.

Proposition 5.1. Theorem 1.1 holds for del Pezzo surfaces of degree 1.

We start with the following.

Lemma 5.2. Let $X$ be a Du Val del Pezzo surface of degree 1. Then $X$ does not admit effective actions of the group $\mathbb{G}_{\mathrm{a}}$.

Proof. Suppose that $X$ admit an effective $\mathbb{G}_{\mathrm{a}}$-action. Let $\Phi: X \rightarrow \mathbb{P}^{1}$ be the anticanonical map. It is $\operatorname{Aut}(X)$-equivariant, and all its fibers are reduced irreducible curves of arithmetic genus 1. Since $\mathbb{G}_{\mathrm{a}}$ cannot effectively act on a smooth elliptic curve, we conclude that $\mathbb{G}_{\mathrm{a}}$ acts non-trivially on the base of $\Phi$. Thus, there is exactly one $\mathbb{G}_{\mathrm{a}}$-invariant fiber, say $C$. Any fiber of $\Phi$ different from $C$ is a smooth elliptic curve. Thus, we have

$$
\rho(X)+2=\chi(X)=\chi(C)-1,
$$

so that $\chi(C)=\rho(X)+3 \geqslant 4$. But $\chi(C) \leqslant 2$ because $C$ is a curve of arithmetic genus 1 .

Our next step in proving Theorem 1.1 for del Pezzo surfaces of degree 1 is the following.

Lemma 5.3. If $X$ is one of the surfaces $1^{\circ}, 2^{\circ}, 3^{o}, 4^{o}$, then $\operatorname{Aut}^{0}(X) \cong \mathbb{G}_{\mathrm{m}}$.

Proof. By Lemma 5.2, these surfaces do not admit a $\mathbb{G}_{\mathrm{a}}$-action, and they are not toric because their singularities are not cyclic quotients. On the other hand, it is easy to see that each of these surfaces admits a $\mathbb{G}_{\mathrm{m}}$-action.

We also need the following easy local fact. 


\section{Cheltsov and Yu. Prokhorov}

Lemma 5.4. Let $(X \ni P)$ be a $D u$ Val singularity defined over $\mathbb{C}$ that contains a reduced irreducible curve $C$ such that $C$ is a Cartier divisor on $X$ and the singularity $(C \ni P)$ is a simple cusp.

(i) If $(X \ni P)$ is of type $\mathrm{A}_{n}$, then $n \leqslant 2$.

(ii) If $(X \ni P)$ is of type $\mathrm{D}_{n}$ with $n \geqslant 5$, then some small analytic neighborhood of $(X \ni P)$ can be given by the equation

$$
x^{2}+y^{2} z+z^{n-1}=0,
$$

so that $C$ is cut out by $z=y+\phi(x, y, z)$, where $\operatorname{mult}_{0}(\phi) \geqslant 2$.

Proof. Let us prove assertion (i). In a neighborhood of the point $P$, the curve $C$ is cut out by a hypersurface, say $H$. Thus, we have $C=X \cap H$ in $\mathbb{C}^{3}$. Since the multiplicity of the curve $C$ at $P$ equals 2 , the hypersurface $H$ is smooth at $P$. Therefore, we may assume that $H$ is given by $z=0$ and $C$ is given by

$$
\left\{\begin{array}{l}
x^{2}+y^{3}=0, \\
z=0
\end{array}\right.
$$

Hence, the equation of the surface $X$ is

$$
x^{2}+y^{3}+z \phi(x, y, z)=0,
$$

Since $P \in X$ is a point of type $\mathrm{A}_{n}$, the rank of the quadratic part of this equation is at least 2 . Then $\phi(x, y, z)$ contains a linear term. This implies that $P \in X$ is of type $\mathrm{A}_{2}$ or $\mathrm{A}_{3}$.

Now, let us prove assertion (ii). We may assume that $P \in X$ is given in $\mathbb{C}^{3}$ by the equation

$$
x^{2}+y^{2} z+z^{n-1}=0 .
$$

As above, we have $C=X \cap H$, where $H$ is a hypersurface that is smooth at $P$. Then the equation of the hypersurface $H$ must contain a linear term. Moreover, one can see that this equation must be of the form $z=y+\phi(x, y, z)$, which implies assertion (ii).

Corollary 5.5. Let $X$ be a surface admitting an effective $\mathbb{G}_{\mathrm{m}}$-action, let $C$ be a $\mathbb{G}_{\mathrm{m}}$-invariant reduced irreducible curve in $X$ that is a Cartier divisor on $X$, and let $P$ be its singular point. Suppose that $X$ has a $D u$ Val singularity of type $D_{n}$ at $P$ and $C$ has a simple cusp at $P$. Then $n=4$.

Proof. Suppose that $n>4$. There exists a $\mathbb{G}_{\mathrm{m}}$-equavariant embedding of the germ $P \in X$ to $\mathbb{C}^{3}$. Let us choose $\mathbb{G}_{\mathrm{m}}$-semi-invariant coordinates in $\mathbb{C}^{3}$. By Lemma 5.4 , we see that $C=X \cap H$, where the equation of $H$ has the form $z=y+\phi(x, y, z)$. But this equation cannot be $\mathbb{G}_{\mathrm{m}}$-semi-invariant, which gives a contradiction.

Now, we are ready to prove Proposition 5.1.

Proof of Proposition 5.1. Let $X$ be a Du Val del Pezzo surface of degree 1. Then $X$ does not admit an effective $\mathbb{G}_{\mathrm{a}}$-action by Lemma 5.2. Thus, the group $\operatorname{Aut}(X)$ is infinite if and only if $X$ admits an effective action of the group $\mathbb{G}_{\mathrm{m}}$.

Suppose that $X$ admits an effective $\mathbb{G}_{\mathrm{m}}$-action. By Lemma 5.3, to complete the proof, it is enough to show that $X$ is one of the surfaces $1^{\circ}, 2^{\circ}, 3^{\circ}, 4^{\circ}$.

Let $\Phi: X \rightarrow \mathbb{P}^{1}$ be the anticanonical map. It is $\mathbb{G}_{\mathrm{m}}$-equivariant, and all its fibers are reduced irreducible curves of arithmetic genus 1 . Since $\mathbb{G}_{m}$ cannot effectively act on a smooth elliptic 


\section{Del Pezzo surfaces}

curve, we conclude that $\mathbb{G}_{\mathrm{m}}$ acts non-trivially on the base of $\Phi$. There are exactly two $\mathbb{G}_{\mathrm{m}^{-}}$ invariant fibers. Denote them by $C_{1}$ and $C_{2}$. Any fiber of $\Phi$ different from $C_{1}$ and $C_{2}$ is a smooth elliptic curve. Thus, we have

$$
\rho(X)+2=\chi(X)=\chi\left(C_{1}\right)+\chi\left(C_{2}\right)-1,
$$

so that $\chi\left(C_{1}\right)+\chi\left(C_{2}\right)=\rho(X)+3 \geqslant 4$. Since $\chi\left(C_{i}\right) \leqslant 2$, we have $\rho(X)=1$ and $\chi\left(C_{1}\right)=\chi\left(C_{2}\right)=2$. This means, in particular, that $C_{1}$ and $C_{2}$ are cuspidal curves of arithmetic genus 1 .

Let $P_{i}$ be the singular point of $C_{i}$. Then

$$
\varnothing \neq \operatorname{Sing}(X) \subset\left\{P_{1}, P_{2}\right\} .
$$

The singularity of the surface $X$ at the point $P_{1}$ is of type $\mathrm{A}_{n_{1}}, \mathrm{D}_{n_{1}}$, or $\mathrm{E}_{n_{1}}$ for some $n_{1} \geqslant 1$. Likewise, if $X$ is singular at $P_{2}$, then $P_{2}$ is a singular point of type $\mathrm{A}_{n_{2}}, \mathrm{D}_{n_{2}}$, or $\mathrm{E}_{n_{2}}$ for some $n_{2} \geqslant 0$, where $n_{2}=0$ simply means that the point $P_{1}$ is the only singular point of the del Pezzo surface $X$. Without loss of generality, we may assume that $n_{1} \geqslant n_{2}$. Since $\rho(X)=1$, we have that $n_{1}+n_{2}=8$ and $n_{1} \geqslant 4$. Now, using Corollary 5.5, we obtain the following possibilities for Type $(X): \mathrm{E}_{8}, \mathrm{E}_{7} \mathrm{~A}_{1}, \mathrm{E}_{6} \mathrm{~A}_{2}, 2 \mathrm{D}_{4}$. But $X$ is uniquely determined by Type $(X)$ and the fact that $\left|-K_{X}\right|$ has two singular curves that are both cuspidal. This follows from [Ye02, Theorem 1.2] and [Ye02, Table 4.1]; see also Remark B.5. Thus, we conclude that $X$ is one of the surfaces $1^{\circ}$, $2^{\circ}, 3^{\circ}, 4^{o}$ (see, for example, [Bri68, Satz 2.11]).

Let us conclude this section with an observation that the surfaces $2^{\circ}, 3^{\circ}, 4^{\circ}$ can be obtained as finite quotients of other surfaces in Table 3. The surface $2^{\circ}$ is the quotient of the surface $11^{o}$ by $\boldsymbol{\mu}_{2}$. The surface $3^{\circ}$ is the quotient of the cubic surface $22^{\circ}$ by $\boldsymbol{\mu}_{3}$, and $4^{o}$ is the quotient of a special member of the family $35^{\circ}$ by $\boldsymbol{\mu}_{2} \times \boldsymbol{\mu}_{2}$. In all these cases, the action of the group is free outside the singular locus. This observation can be used to obtain the description of the surfaces $2^{\circ}, 3^{\circ}, 4^{\circ}$. To show this, one can look at the exact sequence

$$
0 \longrightarrow \operatorname{Pic}(X) \stackrel{\alpha}{\longrightarrow} \mathrm{Cl}(X) \stackrel{\beta}{\longrightarrow} \bigoplus_{P \in X} \mathrm{Cl}(X, P),
$$

where $\mathrm{Cl}(X, P)$ is the local Weil divisor class group of the point $P \in X$. The map $\alpha$ is a primitive embedding. Hence, we have $\mathrm{Cl}(X)=\operatorname{Pic}(X) \oplus \mathrm{Cl}(X)_{\text {tors }}$. By Corollary B.6, we have

$$
\mathrm{Cl}(X)_{\text {tors }} \neq 0 \text {. }
$$

By Lemma 2.9(ii), the group $\mathrm{Cl}(X)_{\text {tors }}$ defines a Galois abelian cover $\pi: X^{\prime} \rightarrow X$ which is étale outside of the locus $\operatorname{Sing}(X)$ and whose degree is $\left|\mathrm{Cl}(X)_{\text {tors }}\right|$. Using the local description of such covers (see [Rei87, Bri68]), we see that Type $\left(X^{\prime}\right)=\mathrm{E}_{6}, \mathrm{D}_{4}, 2 \mathrm{~A}_{1}$ in the cases $2^{o}, 3^{\circ}, 4^{o}$, respectively.

\section{Del Pezzo surfaces of degree 2}

In this section, we prove Theorem 1.1 for del Pezzo surfaces of degree 2. To do this, we need one (probably known) result about singular cubic and quartic curves (cf. [Hui79, Wal95]).

Proposition 6.1. Let $C$ be a reduced cubic or quartic curve in $\mathbb{P}^{2}$ such that Aut $\left(\mathbb{P}^{2}, C\right)$ is infinite. Then the curve $C$ and the group $\operatorname{Aut}^{0}\left(\mathbb{P}^{2}, C\right)$ are given in Table 2 below: 


\section{Cheltsov and Yu. Prokhorov}

TABLE 2.

\begin{tabular}{|c|c|}
\hline Equation of the curve $C$ up to the action of $\mathrm{PGL}_{3}(\mathbb{k})$ & $\mathrm{Aut}^{0}\left(\mathbb{P}^{2}, C\right)$ \\
\hline$x_{0} x_{1}\left(x_{0}+x_{1}\right)=0$ & $\mathbb{G}_{\mathrm{a}}^{2} \rtimes \mathbb{G}_{\mathrm{m}}$ \\
\hline$x_{0} x_{1} x_{2}=0$ & $\mathbb{G}_{\mathrm{m}}^{2}$ \\
\hline$x_{0}\left(x_{0} x_{2}+x_{1}^{2}\right)=0$ & $\mathbb{B}_{2}$ \\
\hline$x_{0}^{2} x_{2}+x_{1}^{3}=0$ & $\mathbb{G}_{\mathrm{m}}$ \\
\hline$x_{1}\left(x_{0} x_{2}+x_{1}^{2}\right)=0$ & $\mathbb{G}_{\mathrm{m}}$ \\
\hline$x_{0}\left(x_{0}^{2} x_{2}+x_{1}^{3}\right)=0$ & $\mathbb{B}_{2}$ \\
\hline$x_{0} x_{1}\left(x_{0} x_{2}+x_{1}^{2}\right)=0$ & $\mathbb{G}_{\mathrm{m}}$ \\
\hline$\left(x_{0} x_{2}+x_{1}^{2}\right)^{2}-x_{0}^{4}=0$ & $\mathbb{G}_{\mathrm{m}}$ \\
\hline$x_{2}\left(x_{0}^{2} x_{2}+x_{1}^{3}\right)=0$ & $\mathbb{G}_{\mathrm{a}}$ \\
\hline$x_{0} x_{1} x_{2}\left(x_{1}+x_{2}\right)=0$ & $\mathbb{G}_{\mathrm{m}}$ \\
\hline$x_{0} x_{1}\left(x_{0} x_{1}+x_{2}^{2}\right)=0$ & $\mathbb{G}_{\mathrm{m}}$ \\
\hline$x_{0}^{3} x_{2}+x_{1}^{4}=0$ & $\mathbb{G}_{\mathrm{m}}$ \\
\hline$x_{1}\left(x_{0}^{2} x_{2}+x_{1}^{3}\right)=0$ & $\mathbb{G}_{\mathrm{m}}$ \\
\hline$\left(x_{0}-\lambda x_{1}\right)=\mathbb{k} \backslash\{0,1\}$ & $\mathbb{G}_{\mathrm{m}}$ \\
\hline$\left.x_{2}^{2}+\lambda x_{0} x_{1}\right)=0$ for $\lambda \in \mathbb{k} \backslash\{0,1\}$ & $\mathbb{G}_{\mathrm{m}}$ \\
\hline
\end{tabular}

Proof. If $C$ is one of the curves in Table 2, we can explicitly describe $\mathrm{Aut}^{0}\left(\mathbb{P}^{2}, C\right)$ by finding all elements in Aut $\left(\mathbb{P}^{2}\right) \cong \mathrm{PGL}_{3}(\mathbb{k})$ that leave every irreducible component of the curve $C$ invariant. For example, if $C$ is given by $x_{0} x_{1}\left(x_{0}+x_{1}\right)=0$, then $\operatorname{Aut}^{0}\left(\mathbb{P}^{2}, C\right)$ consists of the transformations

$$
\left(x_{0}: x_{1}: x_{2}\right) \longmapsto\left(t x_{0}: t x_{1}: x_{2}+a x_{0}+b x_{1}\right)
$$

for any $t \in \mathbb{k}^{*}, a \in \mathbb{k}$, and $b \in \mathbb{k}$. Thus, in this case, we have Aut ${ }^{0}\left(\mathbb{P}^{2}, C\right) \cong \mathbb{G}_{\mathrm{a}}^{2} \rtimes \mathbb{G}_{\mathrm{m}}$, as required.

Similarly, if $C$ is given by $x_{0}\left(x_{0} x_{2}+x_{1}^{2}\right)=0$, then $\mathrm{Aut}^{0}\left(\mathbb{P}^{2}, C\right)$ consists of the transformations

$$
\left(x_{0}: x_{1}: x_{2}\right) \longmapsto\left(t^{2} x_{0}: t x_{1}+a x_{0}: x_{2}-\frac{2 a}{t} x_{1}-\frac{a^{2}}{t^{2}} x_{0}\right)
$$

for any $t \in \mathbb{k}^{*}$ and $a \in \mathbb{k}$, so that $\operatorname{Aut}^{0}\left(\mathbb{P}^{2}, C\right) \cong \mathbb{G}_{\mathrm{a}} \rtimes(1) \mathbb{G}_{\mathrm{m}}$. Likewise, if $C$ is the cubic $x_{0}^{2} x_{2}+x_{1}^{3}=0$, then $\operatorname{Aut}^{0}\left(\mathbb{P}^{2}, C\right)$ consists of the transformations $\left(x_{0}: x_{1}: x_{2}\right) \mapsto\left(t^{3} x_{0}: t^{2} x_{1}: x_{2}\right)$, where $t \in \mathbb{k}^{*}$. Thus, in this case, we have $\operatorname{Aut}^{0}\left(\mathbb{P}^{2}, C\right) \cong \mathbb{G}_{\mathrm{m}}$.

The computations are very similar in all remaining cases. For instance, if $C$ is the quartic curve that is given by $\left(x_{0} x_{2}+x_{1}^{2}\right)^{2}-x_{0}^{4}=0$, then Aut $\left(\mathbb{P}^{2}, C\right)$ consists of the transformations

$$
\left(x_{0}: x_{1}: x_{2}\right) \longmapsto\left(\zeta^{2} x_{0}: \zeta x_{1}-\frac{\zeta a}{2} x_{0}: x_{2}+a x_{1}-\frac{a^{2}}{4} x_{0}\right),
$$

where $\zeta \in\{ \pm 1, \pm i\}$ and $a \in \mathbb{k}$, which implies that $\operatorname{Aut}\left(\mathbb{P}^{2}, C\right) \cong \mathbb{G}_{\mathrm{a}} \rtimes \boldsymbol{\mu}_{4}$, so that $\operatorname{Aut}^{0}\left(\mathbb{P}^{2}, C\right) \cong \mathbb{G}_{\mathrm{a}}$. We leave the computations in the remaining cases to the reader.

Therefore, to complete the proof, we must show that $C$ is one of the curves listed in Table 2 . If $C$ is the cubic curve, then $C$ must be singular. On the other hand, all singular cubic curves 


\section{Del Pezzo surfaces}

are already listed in Table 2 except for the nodal one that is given by

$$
x_{2}\left(x_{0}^{2}+x_{1}^{2}\right)+x_{1}^{2}=0 .
$$

However, if $C$ is this curve, then Aut $\left(\mathbb{P}^{2}, C\right)$ is finite. Therefore, we may assume that $\operatorname{deg}(C)=4$. Then we may have the following five cases:

(i) the curve $C$ is irreducible;

(ii) $C=C_{1}+C_{2}$, where $C_{1}$ is a line and $C_{2}$ is an irreducible cubic;

(iii) $C=C_{1}+C_{2}$, where $C_{1}$ and $C_{2}$ are irreducible conics;

(iv) $C=C_{1}+C_{2}+C_{3}$, where $C_{1}$ and $C_{2}$ are lines and $C_{3}$ is an irreducible conic;

(v) $C=C_{1}+C_{2}+C_{3}+C_{3}$, where $C_{1}, C_{2}, C_{3}$, and $C_{4}$ are lines.

Moreover, by our assumption, the group Aut $\left(\mathbb{P}^{2}, C\right)$ contains a subgroup isomorphic to either $\mathbb{G}_{\mathrm{a}}$ or $\mathbb{G}_{\mathrm{m}}$ (or both). We deal with these two (slightly overlapping) possibilities separately.

Suppose that Aut $\left(\mathbb{P}^{2}, C\right) \supset \mathbb{G}_{\mathrm{a}}$. Since each irreducible component is $\mathbb{G}_{\mathrm{a}}$-invariant, we conclude that case (ii) is impossible. Likewise, if $C$ is irreducible, then $C$ has a $\mathbb{G}_{\mathrm{a}}$-open orbit $U \cong \mathbb{A}^{1}$. Hence, the curve $C$ must be rational, its normalization morphism must be a homeomorphism, and the complement $C \backslash U$ is a single point, say $P$. The projection $C \rightarrow \mathbb{P}^{1}$ from $P$ must be $\mathbb{G}_{\mathrm{a}}$-equivariant, so it is an isomorphism on $U$. This implies that $P$ is a triple point and that there is exactly one line $L \subset \mathbb{P}^{2}$ such that $C \cap L=P$. We may assume that $P=(0: 0: 1)$ and that $L$ is given by $x_{0}=0$. Then the equation of $C$ has the form $x_{0}^{3} x_{2}+x_{1}^{4}=0$. But then $\operatorname{Aut}^{0}\left(\mathbb{P}^{2}, C\right) \cong \mathbb{G}_{\mathrm{m}}$, which gives a contradiction. Hence, we conclude that case (i) is also impossible.

If we are in case (iii), then the $\mathbb{G}_{\mathrm{a}}$-action of each irreducible conic $C_{1}$ and $C_{2}$ is effective, so that the intersection $C_{1} \cap C_{2}$ consists of one point. In this case, in appropriate projective coordinates, the curve $C$ is given by

$$
\left(x_{1} x_{2}+x_{0}^{2}+x_{1}^{2}\right)\left(x_{1} x_{2}+x_{0}^{2}-x_{1}^{2}\right)=0,
$$

so that $C$ is listed in Table 2 , as required.

If we are in case (iv) or (v), then the closure of any one-dimensional $\mathbb{G}_{\text {a }}$-orbit is a line in the pencil generated by $C_{1}$ and $C_{2}$, which implies that $C$ is a union of four lines passing through one point. Hence, we are in case (v), and the curve $C$ can be given by

$$
x_{0} x_{1}\left(x_{0}-x_{1}\right)\left(x_{0}-\lambda x_{1}\right)=0
$$

for some $\lambda \in \mathbb{k} \backslash\{0,1\}$, so that $C$ is in Table 2 as well.

To complete the proof, we may assume that Aut $\left(\mathbb{P}^{2}, C\right) \supset \mathbb{G}_{\mathrm{m}}$. If $C$ is irreducible, then it can be given as the closure of the image of the map $t \mapsto\left(1: t: t^{4}\right)$, so that $C$ is the curve $x_{0}^{3} x_{2}-x_{1}^{4}=0$ in Table 2. Hence, we may assume that $C$ is reducible; that is, we are not in case (i).

Suppose that $\mathbb{G}_{\mathrm{m}}$ acts trivially on some irreducible component of the curve $C$. This component must be a line, so that we are in one of the cases (ii), (iv), or (v). Without loss of generality, we may assume that $\mathbb{G}_{\mathrm{m}}$ acts trivially on the line $C_{1}$. Then there exists a $\mathbb{G}_{\mathrm{m}}$-fixed point $O \in \mathbb{P}^{2} \backslash C_{1}$, so that the closure of any $\mathbb{G}_{\mathrm{m}}$-orbit in $\mathbb{P}^{2}$ is a line connecting $O$ and a point in $C_{1}$. This implies that we are in case (v), and the lines $C_{2}, C_{3}, C_{4}$ all pass through $O$, so that $C$ is given by

$$
x_{0} x_{1} x_{2}\left(x_{1}+x_{2}\right)=0
$$

in appropriate projective coordinates. This curve is in Table 2. Therefore, to complete the proof, we may assume that $\mathbb{G}_{\mathrm{m}}$ acts effectively on each irreducible component of the curve $C$. 


\section{Cheltsov and Yu. Prokhorov}

If we are in case (v), then each line among $C_{1}, C_{2}, C_{3}$, and $C_{4}$ meets the union of the remaining lines in at most two points. This implies that all these four lines must pass through one point. Such a curve is in Table 2, and we already came across it earlier in the proof. Hence, case (v) is done.

Suppose that we are in case (iii), so that $C=C_{1}+C_{2}$, where both $C_{1}$ and $C_{2}$ are irreducible conics. Then the intersection $C_{1} \cap C_{2}$ consists of at most two points. Moreover, the intersection cannot consist of one point since otherwise we would have Aut $\left(\mathbb{P}^{2}, C\right) \cong \mathbb{G}_{\mathrm{a}}$. Hence, we see that the intersection $C_{1} \cap C_{2}$ consists of exactly two points. Then the curve $C$ can be given by

$$
\left(x_{2}^{2}+x_{0} x_{1}\right)\left(x_{2}^{2}+\lambda x_{0} x_{1}\right)=0
$$

for some $\lambda \in \mathbb{k} \backslash\{0,1\}$. This curve is also in Table 2 . Thus, case (iii) is also done.

Now we suppose that we are in case (iv). Then $C_{1}$ and $C_{2}$ are lines, and $C_{3}$ is a conic. Then

$$
\#\left(C_{3} \cap\left(C_{1} \cup C_{2}\right)\right) \leqslant 2,
$$

so that at least one of the lines $C_{1}$ and $C_{2}$ must be tangent to $C_{3}$. If only one of them is tangent, then $C$ can be given by $x_{0} x_{1}\left(x_{0} x_{2}+x_{1}^{2}\right)=0$. Similarly, if both lines are tangent to the conic $C_{3}$, then $C$ can be given by $x_{0} x_{1}\left(x_{0} x_{1}+x_{2}^{2}\right)=0$. In both subcases, the curve $C$ is in Table 2 .

Finally, we suppose that we are in case (ii). Then $C_{2}$ is a cuspidal cubic curve. Now, choosing appropriate coordinates on $\mathbb{P}^{2}$, we may assume that $C_{2}$ is given by

$$
x_{0}^{2} x_{2}-x_{1}^{3}=0,
$$

and the $\mathbb{G}_{\mathrm{m}}$-action on $\mathbb{P}^{2}$ is described earlier in the proof. Then the $\mathbb{G}_{\mathrm{m}}$-action on $C_{2}$ has exactly two fixed points: the points $(0: 0: 1)$ and $(1: 0: 0)$. If the line $C_{1}$ passes through both of them, then the curve $C$ is given by

$$
x_{0} x_{1}\left(x_{0} x_{2}+x_{1}^{2}\right)=0
$$

Similarly, if $(1: 0: 0) \in C_{1}$ and $(0: 0: 1) \notin C_{1}$, then the curve $C$ is given by $x_{2}\left(x_{0}^{2} x_{2}+x_{1}^{3}\right)=0$. Vice versa, if $(1: 0: 0) \notin C_{1}$ and $(0: 0: 1) \in C_{1}$, then the curve $C$ is given by $x_{0}\left(x_{0}^{2} x_{2}+x_{1}^{3}\right)=0$. In every subcase, we see that the quartic curve $C$ is listed in Table 2 , as required.

Using Proposition 6.1, we immediately obtain the following.

Corollary 6.2. Theorem 1.1 holds for del Pezzo surfaces of degree 2.

Proof. Let $X$ be a Du Val del Pezzo surface of degree 2 . Then $X$ is a hypersurface in $\mathbb{P}(1,1,1,2)$ that is given by $w^{2}=\phi_{4}\left(x_{0}, x_{1}, x_{2}\right)$, where $\phi_{4}\left(x_{0}, x_{1}, x_{2}\right)$ is a homogeneous polynomial of degree 4 . The natural projection to $\mathbb{P}^{2}$ gives a double cover $\pi: X \rightarrow \mathbb{P}^{2}$. Let $B$ be the branch curve of this double cover. Then $B$ is the quartic curve in $\mathbb{P}^{2}$ that is given by $\phi_{4}\left(x_{0}, x_{1}, x_{2}\right)=0$.

Since the double cover $\pi$ is $\operatorname{Aut}(X)$-equivariant, it gives a homomorphism $\operatorname{Aut}^{0}(X) \rightarrow$ Aut $^{0}\left(\mathbb{P}^{2}, B\right)$, whose kernel is either trivial or isomorphic to $\boldsymbol{\mu}_{2}$. Thus, the curve $B$ must be one of the quartic curves listed in Table 2 in Proposition 6.1, except for the quartic curve consisting of four lines passing through one point because $X$ has Du Val singularities. Now, going through the equations listed in Table 2 , we obtain all possibilities for the polynomial $\phi_{4}\left(x_{0}, x_{1}, x_{2}\right)$. This shows that if $\operatorname{Aut}(X)$ is infinite, then

- either $\operatorname{Aut}(X) \cong \mathbb{G}_{\mathrm{a}}$ and $X$ is the surface $7^{\circ}$,

- or $\operatorname{Aut}(X) \cong \mathbb{G}_{\mathrm{m}}$ and $X$ is one of the surfaces $5^{\circ}, 6^{\circ}, 8^{\circ}, 9^{\circ}, 10^{\circ}, 11^{\circ}, 12^{\circ}, 13^{\circ}$.

Vice versa, if $X$ is the surface $7^{\circ}$, then the group $\mathbb{G}_{\mathrm{a}}$ acts on $X$ as follows:

$$
\left(x_{0}: x_{1}: x_{2}: w\right) \longmapsto\left(x_{0}+t x_{1}: x_{1}: x_{2}-2 t x_{0}-t^{2} x_{1}: w\right),
$$




\section{Del Pezzo surfaces}

where $t \in \mathbb{G}_{\mathrm{a}}$. Thus, in this case, we have $\operatorname{Aut}(X) \cong \mathbb{G}_{\mathrm{a}}$. Similarly, if $X$ is one of the del Pezzo surfaces $5^{\circ}, 6^{\circ}, 8^{\circ}, 9^{\circ}, 10^{\circ}, 11^{\circ}, 12^{\circ}, 13^{\circ}$, then $X$ admits an effective action of the group $\mathbb{G}_{\mathrm{m}}$, so that $\operatorname{Aut}(X) \cong \mathbb{G}_{\mathrm{m}}$, as listed in Table 3 .

\section{Cubic surfaces}

Now, we prove Theorem 1.1 for del Pezzo surfaces of degree 3, which easily follows from [Sak10]. Nevertheless, we prefer to give an independent proof here.

Lemma 7.1. Let $X$ be a Du Val cubic surface in $\mathbb{P}^{3}$.

(i) If $X$ contains three lines $L_{1}, L_{2}, L_{3}$ that meet one another at three distinct points (a triangle), then $X$ does not admit an effective action of the group $\mathbb{G}_{\mathrm{a}}$.

(ii) If $X$ is toric, then $\rho(X)=1, \#(X)=3$, and the toric boundary is composed of three lines forming a triangle.

Proof. If $X$ contains a triangle and admits an effective $\mathbb{G}_{\mathrm{a}}$-action, then the $\mathbb{G}_{\mathrm{a}}$-action is trivial on the triangle, so that this action is trivial on the hyperplane in $\mathbb{P}^{3}$ that passes through the triangle, which implies that the closure of any $\mathbb{G}_{\mathrm{a}}$-orbit in $X$ is contained in a line. The latter is impossible since $X$ contains finitely many lines. This proves assertion (i)

To prove assertion (ii), suppose that the surface $X$ is toric. Let $D=D_{1}+\cdots+D_{r}$ be the toric boundary. Then $r=\rho(X)+2$. Since every line on $X$ is torus-invariant and $D \sim-K_{X}$, we have

$$
3=\sum_{i=1}^{r}\left(-K_{X}\right) \cdot D_{i} \geqslant r .
$$

Therefore, we conclude that $\rho(X)=1, r=3$, and $-K_{X} \cdot D_{1}=-K_{X} \cdot D_{2}=-K_{X} \cdot D_{3}=1$. Moreover, the lines $D_{1}, D_{2}, D_{3}$ form a triangle because the pair $(X, D)$ has log canonical singularities.

Now, we are ready to prove the following.

Proposition 7.2. Theorem 1.1 holds for weakly minimal cubic surfaces.

Proof. Let $X$ be a weakly minimal Du Val cubic surface. If $\tau(X)=1$, then Theorem 3.12 implies that $X$ is the surfaces $19^{\circ}$, and its basic properties are described in Example 3.11. In this case, the surface $X$ admits an algebraic torus action

$$
\left(x_{0}, x_{1}, x_{2}, x_{3}\right) \longmapsto\left(x_{0}, t x_{1}, t^{2} x_{2}, t^{-1} x_{3}\right) .
$$

Since $X$ contains three lines passing through one point, it is not toric. Since $X$ contains a triangle, it does not admit a unipotent group action by Lemma 7.1, so that $\operatorname{Aut}^{0}(X) \cong \mathbb{G}_{\mathrm{m}}$, as required.

Thus, to complete the proof, we may assume that $\tau(X)>1$. By Theorem 3.8, we have only the following possibilities: $14^{\circ}, 15^{\circ}, 16^{\circ}, 18^{\circ}, 21^{\circ}, 23^{\circ}$.

Consider the cases $16^{\circ}, 21^{\circ}, 23^{\circ}$. From the equations in Theorem 3.8, we see that $X$ contains a triangle that is cut out by $y_{1} y_{2}=0$. Then by Lemma 7.1(i), we conclude that the unipotent radical of $\operatorname{Aut}^{0}(X)$ is trivial. The surface $16^{\circ}$ is a toric cubic surface because its equation is binomial, and the surfaces $21^{\circ}$ and $23^{\circ}$ are not toric by Lemma 7.1(ii). Therefore, if $X$ is the surface $16^{\circ}$, then $\operatorname{Aut}^{0}(X) \cong \mathbb{G}_{\mathrm{m}}^{2}$. Similarly, if $X$ is one of the surfaces $21^{\circ}$ or $23^{\circ}$, then we have $\operatorname{Aut}^{0}(X) \cong \mathbb{G}_{\mathrm{m}}$ because $X$ admits a diagonal effective action of the group $\mathbb{G}_{\mathrm{m}}$. 


\section{Cheltsov and Yu. Prokhorov}

Now, we suppose that $X$ is the surface $18^{\circ}$. Then Type $(X)=\mathrm{A}_{5}$, and it follows from Theorem 3.8 that $X$ is a hypersurface in $\mathbb{P}(1,2,3,3)$ that is given by

$$
y_{3}^{2}=y_{2}^{3}+y_{1}^{6}+y_{1} y_{2} y_{3}^{\prime} \text {. }
$$

Let $L_{1}, L_{2}, L_{3}$ be the curves $y_{1}=y_{3}^{2}-y_{2}^{3}=0, y_{2}=y_{3}-y_{1}^{3}=0, y_{2}=y_{3}+y_{1}^{3}=0$, respectively. Then $L_{1}, L_{2}$, and $L_{3}$ are lines meeting at one point. If $X$ admits an effective action of the group $\mathbb{G}_{\mathrm{m}}$, then $L_{3}$ contains a $\mathbb{G}_{\mathrm{m}}$-fixed point $P \notin \operatorname{Sing}(X)$ and there exists a $\mathbb{G}_{\mathrm{m}}$-equivariant diagram

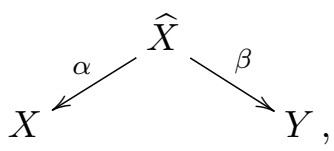

where $\alpha$ is the blow-up of the point $P$, the morphism $\beta$ is the birational contraction of the proper transform of the line $L_{3}$, and $Y$ is a singular del Pezzo surface of degree 2 such that $\operatorname{Type}(X)=\mathrm{A}_{6}$. The latter contradicts Corollary 6.2 , so that $X$ does not admit an effective action of the group $\mathbb{G}_{\mathrm{m}}$. Then $\operatorname{Aut}^{0}(X)$ is unipotent. By Corollary 2.11, the surface $X$ does not admit an effective $\mathbb{G}_{\mathrm{a}}^{2}$-action. Then $\operatorname{dim} \operatorname{Aut}^{0}(X) \leqslant 1$. On the other hand, the group $\operatorname{Aut}(X)$ contains the transformations

$$
\left(y_{1}: y_{2}: y_{3}: y_{3}^{\prime}\right) \longmapsto\left(y_{1}: y_{2}: y_{3}+a y_{1} y_{2}: y_{3}^{\prime}+2 a y_{3}+a^{2} y_{1} y_{2}\right)
$$

where $a \in \mathbb{k}$. They generate a group isomorphic to $\mathbb{G}_{\mathrm{a}}$. Then $\operatorname{Aut}^{0}(X) \cong \mathbb{G}_{\mathrm{a}}$ by Corollary 2.2 .

Let $X$ be the surface $15^{\circ}$. As in the previous case, the surface $X$ is a sextic hypersurface in the weighted projective space $\mathbb{P}(1,2,3,3)$. But now the surface $X$ is given by $y_{3}^{2}=y_{2}^{3}+y_{3}^{\prime} y_{1} y_{2}$. Observe that the group $\operatorname{Aut}(X)$ contains the transformations

$$
\left(y_{1}: y_{2}: y_{3}: y_{3}^{\prime}\right) \longmapsto\left(y_{1}: t^{2} y_{2}: t^{3} y_{3}+a t^{3} y_{1} y_{2}: t^{4} y_{3}^{\prime}+2 a t^{4} y_{3}+a^{2} t^{4} y_{1} y_{2}\right)
$$

for any $a \in \mathbb{k}$ and $t \in \mathbb{k}^{*}$. They generate a group isomorphic to $\mathbb{B}_{2}$. This implies that $\operatorname{Aut}^{0}(X) \cong \mathbb{B}_{2}$ because $X$ is not toric by Lemma 7.1(ii) and $X$ admits no effective $\mathbb{G}_{\mathrm{a}}^{2}$-action by Corollary 2.11.

Finally, if $X$ is the surface $14^{\circ}$, then it follows from Theorem 3.8 that $X$ is a hypersurface in $\mathbb{P}(1,2,3,3)$ that is given by $y_{3}^{2}=y_{2}^{3}+y_{3}^{\prime} y_{1}^{3}$. Using this, one can show that the group $\operatorname{Aut}^{0}(X)$ consists of the transformations

$$
\left(y_{1}: y_{2}: y_{3}: y_{3}^{\prime}\right) \longmapsto\left(y_{1}: t^{2} y_{2}: t^{3} y_{3}+a y_{1}^{3}: t^{6} y_{3}^{\prime}+a^{2} y_{1}^{3}+2 a t^{3} y_{3}\right)
$$

where $a \in \mathbb{k}$ and $t \in \mathbb{k}^{*}$. Thus, in this case, we have $\operatorname{Aut}^{0}(X) \cong \mathbb{G}_{\mathrm{a}} \rtimes_{(3)} \mathbb{G}_{\mathrm{m}}$, which also follows from Corollary B.8.

To complete the proof of Theorem 1.1 for Du Val cubic surfaces, we need the following.

Lemma 7.3. Let $X$ be a non-weakly minimal Du Val cubic surface such that $\operatorname{Aut}^{0}(X)$ is infinite. Then $\operatorname{Aut}^{0}(X) \cong \mathbb{G}_{\mathrm{m}}$ and $X$ is one of the surfaces $17^{\circ}, 20^{\circ}$ or $22^{\circ}$.

Proof. The surface $X$ contains a line $L$ such that $L \subset X \backslash \operatorname{Sing}(X)$. By Lemma 2.16(i), there is a conic bundle $\psi: X \rightarrow \mathbb{P}^{1}$ such that $L$ is its double section. If $X$ admits an effective $\mathbb{G}_{\mathrm{a}}$-action, then the group $\mathbb{G}_{\mathrm{a}}$ fixes the ramification points of the double cover $L \rightarrow \mathbb{P}^{1}$ induced by $\psi$, so that the group $\mathbb{G}_{\mathrm{a}}$ acts trivially on $L$, which implies that it also acts trivially on the fibers of the conic bundle $\psi$, so that the $\mathbb{G}_{\mathrm{a}}$-action on $X$ is trivial, which gives a contradiction. Hence, we conclude that the group $\operatorname{Aut}^{0}(X)$ contains no unipotent subgroups. Then $\operatorname{Aut}^{0}(X)$ must be a torus, which implies that $\operatorname{Aut}^{0}(X) \cong \mathbb{G}_{\mathrm{m}}$ because $X$ is not toric by Lemma 7.1(ii). 


\section{Del Pezzo surfaces}

Let $\psi^{\prime}: X \rightarrow X^{\prime}$ be the contraction of the line $L$. Then $X^{\prime}$ is a quartic Du Val del Pezzo surface such that $\rho\left(X^{\prime}\right)=\rho(X)-1$ and Type $(X)=$ Type $\left(X^{\prime}\right)$. Note that the group $\operatorname{Aut}\left(X^{\prime}\right)$ is infinite, and $\operatorname{Aut}^{0}(X)$ is the stabilizer in $\operatorname{Aut}^{0}\left(X^{\prime}\right)$ of the point $\psi^{\prime}(L)$. Let $U^{\prime}$ be the complement in $X^{\prime}$ of the union of all lines. Then $\psi^{\prime}(L) \in U^{\prime}$ by Corollary 2.14.

Suppose that $\rho(X)=2$. Then $\psi$ is an extremal contraction. By Lemma 2.12(ii), the singular points of the surface $X$ can be of types $\mathrm{D}_{4}, \mathrm{D}_{5}, \mathrm{~A}_{3}$, and $\mathrm{A}_{1}$, where $\mathrm{A}_{1}$ appears an even number of times. We have two possibilities: Type $(X)=\mathrm{D}_{5}$ and $\mathrm{A}_{3} 2 \mathrm{~A}_{1}$, so that $X^{\prime}$ is one of the surfaces $24^{\circ}$ or $25^{\circ}$. In both cases, the subset $U^{\prime}$ is the open Aut ${ }^{0}\left(X^{\prime}\right)$-orbit (cf. Remark B.4), which immediately implies that $\operatorname{Aut}^{0}(X) \cong \mathbb{G}_{\mathrm{m}}$ and $X$ is one of the surface $17^{\circ}$ or $20^{\circ}$.

Now, we assume that $\rho(X)>2$. If Type $(X)=\mathrm{D}_{4}$, then $X^{\prime}$ is the surface $26^{\circ}$. Arguing as above, we see that $\operatorname{Aut}^{0}(X) \cong \mathbb{G}_{\mathrm{m}}$ and $X$ is the surface $22^{\circ}$. Thus, to complete the proof, we may assume that $\rho(X)>2$ and all the singularities of $X$ are of type $\mathrm{A}_{n}$.

We claim that the action of $\operatorname{Aut}^{0}(X)$ on $L$ is trivial. Indeed, suppose that this is not the case. Let us seek a contradiction. Let $P_{1}$ and $P_{2}$ be the ramification points of the double cover $L \rightarrow \mathbb{P}^{1}$, and let $F_{1}$ and $F_{2}$ be the fibers of the conic bundle $\psi$ passing through the points $P_{1}$ and $P_{2}$, respectively. Then $P_{1}$ and $P_{2}$ are fixed by $\operatorname{Aut}^{0}(X)$, and these are all $\operatorname{Aut}^{0}(X)$-fixed points on $L$, so that all fibers of the conic bundle $\psi$ other than $F_{1}$ and $F_{2}$ are smooth. Since $\rho(X)>2$, there exists at least one reducible fiber. Thus, we may assume that $F_{1}$ is reducible. Then $F_{1}=F_{1}^{\prime}+F_{1}^{\prime \prime}$, where $F_{1}^{\prime}$ and $F_{1}^{\prime \prime}$ are lines. Then $P_{1}=F_{1}^{\prime} \cap F_{1}^{\prime \prime} \cap L$, and the surface $X$ is smooth along $F_{1}$, which implies that $\operatorname{Sing}(X) \subset F_{2}$ and $F_{2}$ is irreducible (but multiple). In particular, we have $\rho(X)=3$. On the other hand, using Lemma 2.12(ii), we see that either Type $(X)=2 \mathrm{~A}_{1}$ or Type $(X)=\mathrm{A}_{3}$. This contradicts the Noether formula. Therefore, the action of $\operatorname{Aut}^{0}(X)$ on the line $L$ is trivial, so that the action of the group $\operatorname{Aut}^{0}(X)$ on the base of the conic bundle $\psi$ is trivial as well.

Let $M^{\prime}$ be a line in $X^{\prime}$ (it does exist since $\rho\left(X^{\prime}\right)>1$ ), and let $M$ be its proper transform on $X$. Then $\psi^{\prime}(L) \notin M^{\prime}$ by Corollary 2.14, so that $M$ is a line on $X$ which is disjoint from the line $L$. Then $M$ is an $\operatorname{Aut}^{0}(X)$-invariant curve, which is not contained in the fibers of the conic bundle $\psi$ since $\psi$ is given by the projection from $L$. Therefore, if $C$ is a general fiber of $\psi$, then $C$ contains at least three $\operatorname{Aut}^{0}(X)$-fixed points $C \cap(L \cup M)$, so that the $\operatorname{Aut}^{0}(X)$-action on $C$ must be trivial. This implies that the action of $\operatorname{Aut}^{0}(X)$ on $X$ is also trivial, which gives a contradiction.

Combining Proposition 7.2 and Lemma 7.3, we obtain the following.

Corollary 7.4. Theorem 1.1 holds for del Pezzo surfaces of degree 3.

Thus, Theorem 1.1 holds for Du Val del Pezzo surface of degrees 1, 2, 3, 4, 5, 6. This follows from Proposition 5.1 and Corollaries 4.5, 6.2, and 7.4. This completes the proof of Theorem 1.1.

\section{Big table}

Let $X$ be a Du Val del Pezzo surface such that $\operatorname{Aut}(X)$ is infinite. Then the type Type $(X)$, the degree $K_{X}^{2}$, the Picard rank $\rho(X)$, the number of lines $\#(X)$, the Fano-Weil index $\tau(X)$, the group $\operatorname{Aut}^{0}(X)$, and the equation of the surface $X$ are given below in Table 3 . The column No indicates a del Pezzo surface from which $X$ can be obtained by blowing up a smooth point that does not lie on a line. 


\section{Cheltsov and Yu. Prokhorov}

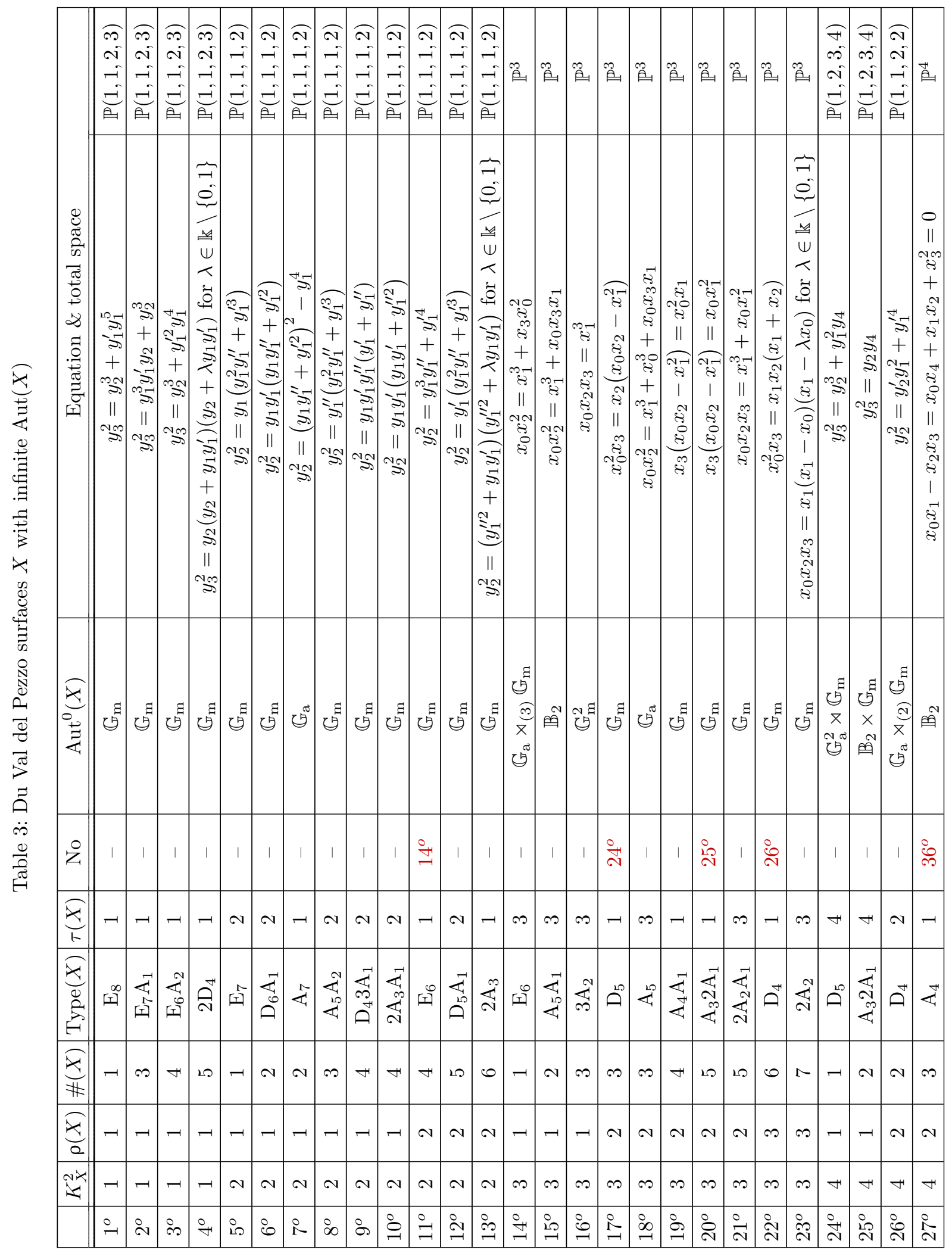




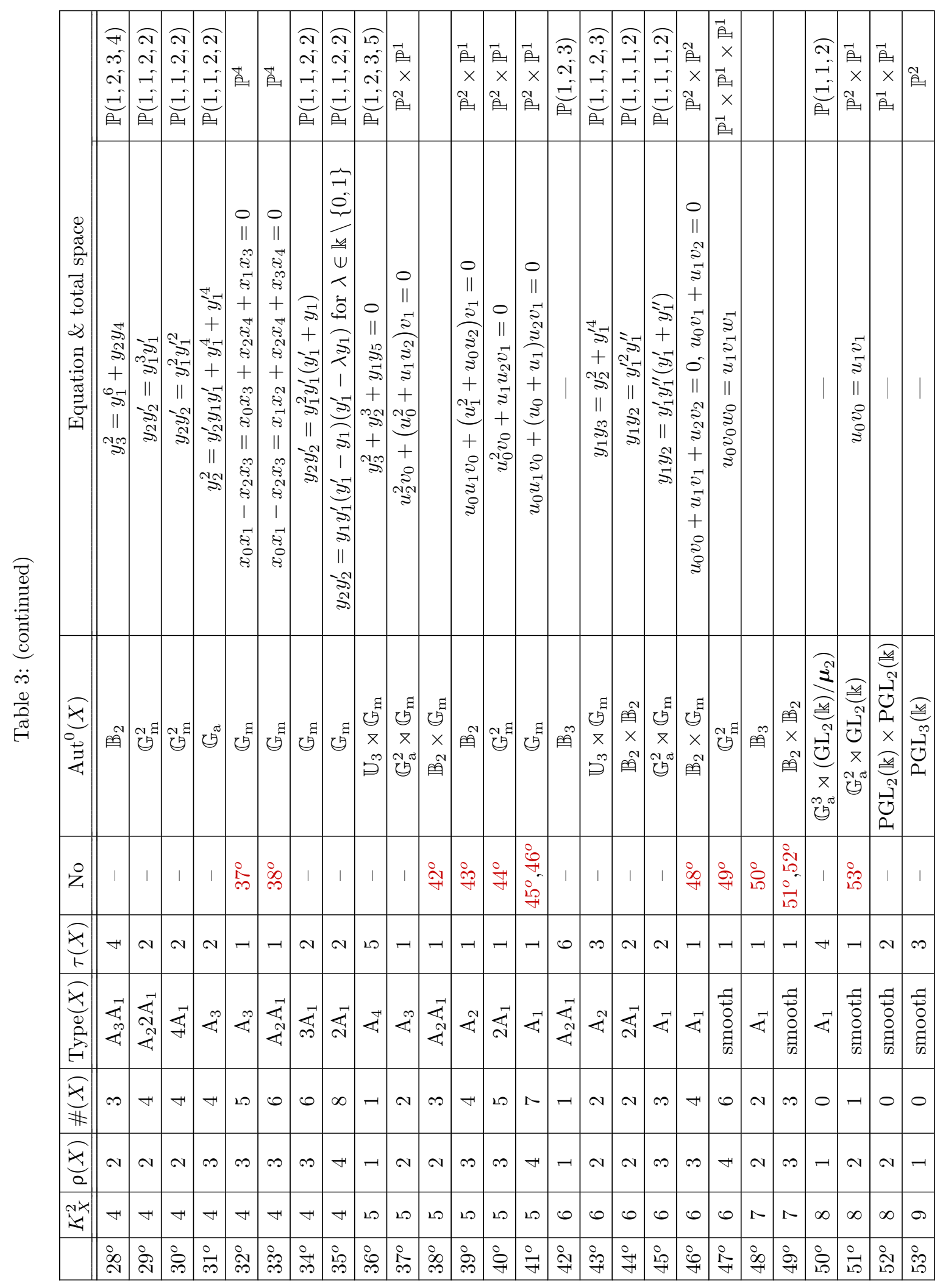




\section{Cheltsov and Yu. Prokhorov}

\section{Appendix A. Lines and dual graphs}

In this appendix, we present equations of the lines on del Pezzo surfaces that appear in Table 3. We also present the dual graphs of all the curves with negative self-intersection numbers on their minimal resolutions. As in the paper [CT88], we denote a (-1)-curve by $\bullet$, and we denote a (-2)-curve by ○. We exclude surfaces $48^{\circ}$ (see Example 2.3), $49^{\circ}, 50^{\circ}, 51^{\circ}, 52^{\circ}, 53^{\circ}$.

Let $X$ be a del Pezzo surface of degree $d$ in Table 3. Take the equation of $X$ from Table 3 . The lines on $X$ and the dual graphs of all the curves with negative self-intersection numbers on its minimal resolution can be described as follows:

$\left(1^{\circ}\right)$ One has $d=1$ and Type $(X)=\mathrm{E}_{8}$. The dual graph is

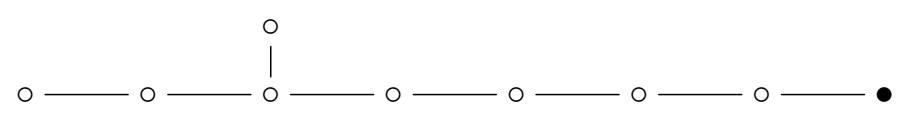

The surface $X$ is weakly minimal. The only line on $X$ is $x_{3}^{2}-x_{2}^{3}=x_{0}=0$.

$\left(2^{\circ}\right)$ One has $d=1$ and $\operatorname{Type}(X)=\mathrm{E}_{7} \mathrm{~A}_{1}$. The dual graph is

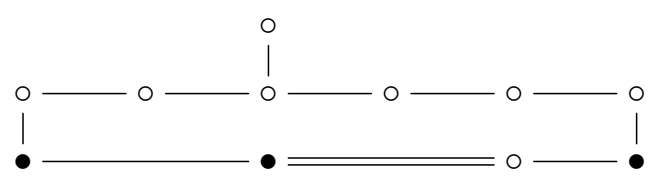

The surface $X$ is weakly minimal. The lines are cut out by $x_{0}=0, x_{1}=0$, and $x_{3}=x_{2}=0$.

$\left(3^{\circ}\right)$ One has $d=1$ and Type $(X)=\mathrm{E}_{6} \mathrm{~A}_{2}$. The dual graph is

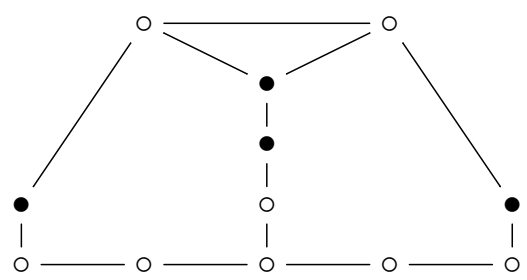

The surface $X$ is weakly minimal. The lines are cut out by $x_{0}=0, x_{1}=0$, and $x_{2}=0$.

$\left(4^{\circ}\right)$ One has $d=1$ and Type $(X)=2 \mathrm{D}_{4}$. The dual graph is

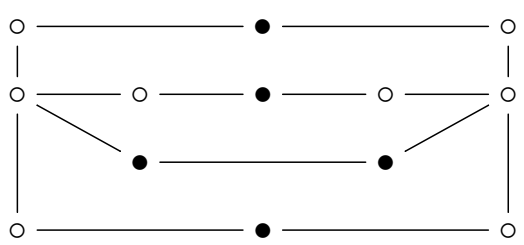

The surface $X$ is weakly minimal. The lines are cut out by $x_{0}=0, x_{1}=0$, and $x_{3}=0$.

$\left(5^{\circ}\right)$ One has $d=2$ and Type $(X)=\mathrm{E}_{7}$. The dual graph is

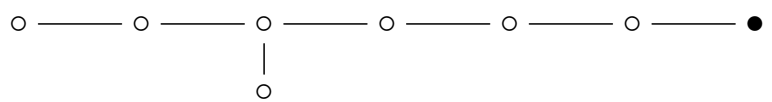

The surface $X$ is weakly minimal, and the line is $x_{0}=x_{3}=0$.

$\left(6^{\circ}\right)$ One has $d=2$ and Type $(X)=\mathrm{D}_{6} \mathrm{~A}_{1}$. The dual graph is

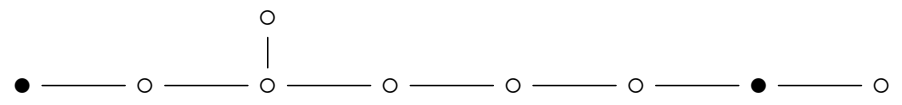

The surface $X$ is weakly minimal. The lines are $x_{0}=x_{3}=0$ and $x_{1}=x_{3}=0$. 


\section{Del Pezzo surfaces}

$\left(7^{\circ}\right)$ One has $d=2$ and Type $(X)=\mathrm{A}_{7}$. The dual graph is

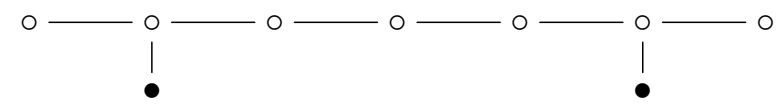

The surface $X$ is weakly minimal, and the lines are $x_{1}=x_{3} \pm x_{0}^{2}=0$.

$\left(8^{\circ}\right)$ One has $d=2$ and Type $(X)=\mathrm{A}_{5} \mathrm{~A}_{2}$. The dual graph is

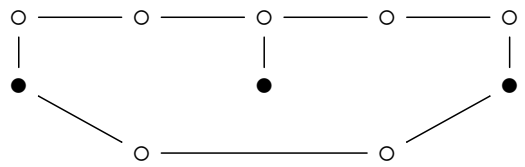

The surface $X$ is weakly minimal. The lines are $x_{1}=x_{3} \pm x_{0} x_{2}=0$ and $x_{2}=x_{3}=0$.

$\left(9^{\circ}\right)$ One has $d=2$ and Type $(X)=\mathrm{D}_{4} 3 \mathrm{~A}_{1}$. The dual graph is

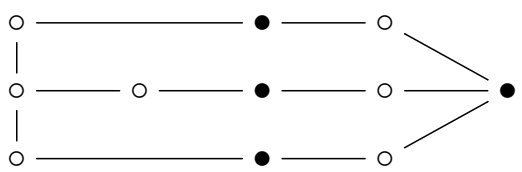

The surface $X$ is weakly minimal. The lines are cut out by $x_{3}=0$.

$\left(10^{\circ}\right)$ One has $d=2$ and Type $(X)=2 \mathrm{~A}_{3} \mathrm{~A}_{1}$. The dual graph is

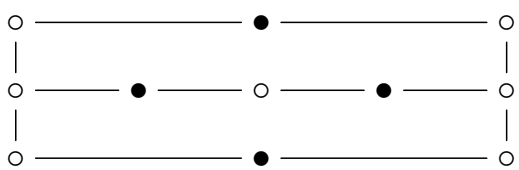

This surface is weakly minimal. The lines are $x_{0}=x_{3}=0, x_{1}=x_{3}=0, x_{2}=x_{3} \pm x_{0} x_{1}=0$.

$\left(11^{\circ}\right)$ One has $d=2$ and $\operatorname{Type}(X)=\mathrm{E}_{6}$. The dual graph is

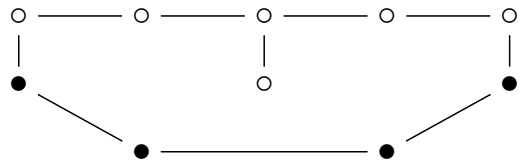

This surface is not weakly minimal. The lines are $x_{0}=x_{3} \pm x_{1}^{2}=0$ and $x_{2}=x_{3} \pm x_{1}^{2}=0$.

$\left(12^{\circ}\right)$ One has $d=2$ and Type $(X)=\mathrm{D}_{5} \mathrm{~A}_{1}$. The dual graph is

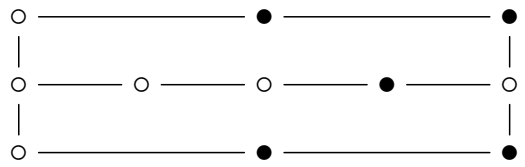

The surface $X$ is weakly minimal. The lines are cut out by $x_{0}=0, x_{2}=0$, and $x_{1}=x_{3}=0$.

$\left(13^{\circ}\right)$ One has $d=2$ and Type $(X)=2 \mathrm{~A}_{3}$. The dual graph is

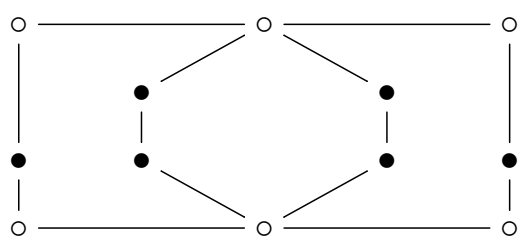

The surface $X$ is weakly minimal. The lines are cut out by $x_{0}=0, x_{1}=0$, and $x_{2}=0$. 


\section{Cheltsov and Yu. Prokhorov}

$\left(14^{\circ}\right)$ One has $d=3$ and Type $(X)=\mathrm{E}_{6}$. The dual graph is

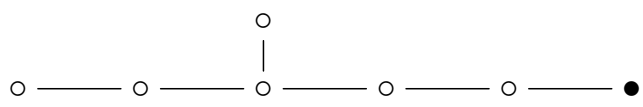

The surface $X$ is weakly minimal. The line is $x_{0}=x_{1}=0$.

$\left(15^{\circ}\right)$ One has $d=3$ and Type $(X)=\mathrm{A}_{5} \mathrm{~A}_{1}$. The dual graph is

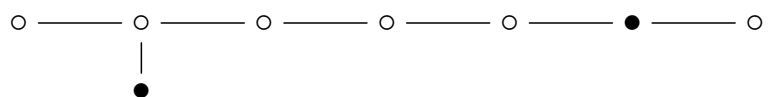

The surface $X$ is weakly minimal. The lines are $x_{0}=x_{1}=0$ and $x_{1}=x_{2}=0$.

$\left(16^{\circ}\right)$ One has $d=3$ and Type $(X)=3 \mathrm{~A}_{2}$. The dual graph is

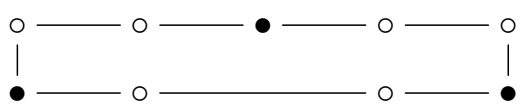

The surface $X$ is weakly minimal. The lines are $x_{0}=x_{3}=0, x_{1}=x_{3}=0$, and $x_{2}=x_{3}=0$.

$\left(17^{\circ}\right)$ One has $d=3$ and Type $(X)=\mathrm{D}_{5}$. The dual graph is

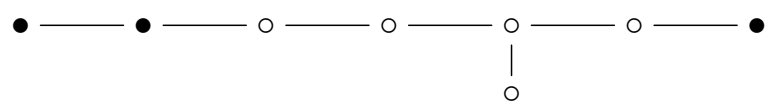

The surface $X$ is weakly minimal. The lines are $x_{0}=x_{1}=0, x_{0}=x_{2}=0$, and $x_{2}=x_{3}=0$.

$\left(18^{\circ}\right)$ One has $d=3$ and Type $(X)=\mathrm{A}_{5}$. The dual graph is

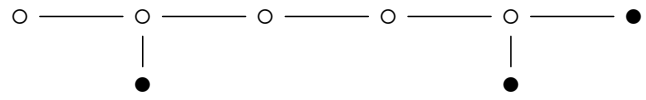

The surface $X$ is weakly minimal. The lines are cut out by $x_{1}=0$.

$\left(19^{\circ}\right)$ One has $d=3$ and Type $(X)=\mathrm{A}_{4} \mathrm{~A}_{1}$. The dual graph is

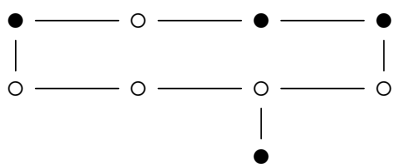

The surface $X$ is weakly minimal. The lines are cut out by $x_{0}=0$ and $x_{1}=0$.

$\left(20^{\circ}\right)$ One has $d=3$ and Type $(X)=\mathrm{A}_{3} 2 \mathrm{~A}_{1}$. The dual graph is

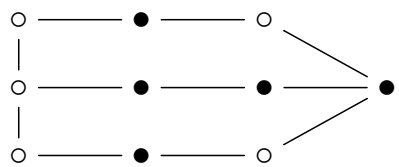

Then $X$ is not weakly minimal. The lines are cut out by $x_{0}=0, x_{1}=0$, and $x_{2}=0$.

$\left(21^{\circ}\right)$ One has $d=3$ and $\operatorname{Type}(X)=2 \mathrm{~A}_{2} \mathrm{~A}_{1}$. The dual graph is

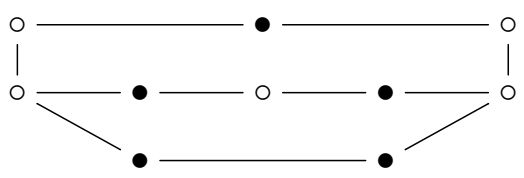

The surface $X$ is weakly minimal. The lines are cut out by $x_{1}=0, x_{2}=0$, and $x_{3}=0$. 


\section{Del Pezzo surfaces}

$\left(22^{\circ}\right)$ One has $d=3$ and Type $(X)=\mathrm{D}_{4}$. The dual graph is

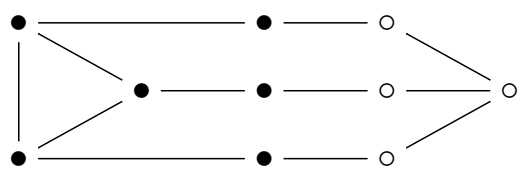

Then $X$ is not weakly minimal. The lines on $X$ are cut by $x_{0}=0$ and $x_{3}=0$.

$\left(23^{\circ}\right)$ One has $d=3$ and Type $(X)=2 \mathrm{~A}_{2}$. The dual graph is

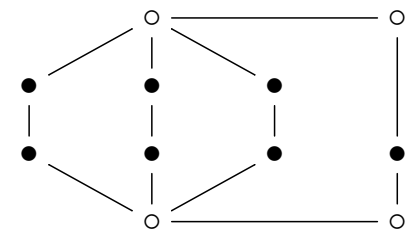

The surface $X$ is weakly minimal. The lines are cut out by $x_{1}=0, x_{2}=0$, and $x_{3}=0$.

$\left(24^{\circ}\right)$ One has $d=4$ and $\operatorname{Type}(X)=\mathrm{D}_{5}$. The dual graph is

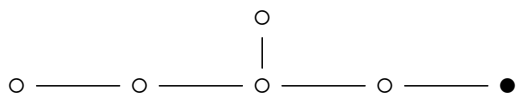

The surface $X$ is weakly minimal. The line is $x_{2}^{2}-x_{1}^{3}=x_{0}=0$.

$\left(25^{\circ}\right)$ One has $d=4$ and $\operatorname{Type}(X)=\mathrm{A}_{3} 2 \mathrm{~A}_{1}$. The dual graph is

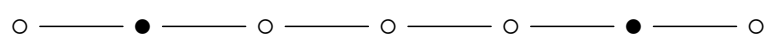

The surface $X$ is weakly minimal. The lines are $x_{1}=x_{2}=0$ and $x_{2}^{2}-x_{1}^{3}=x_{0}=0$.

$\left(26^{\circ}\right)$ One has $d=4$ and Type $(X)=\mathrm{D}_{4}$. The dual graph is

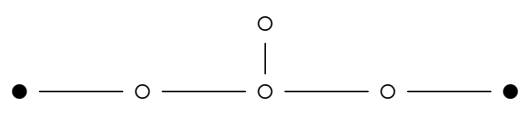

The surface $X$ is weakly minimal, and the lines are $x_{2} \pm x_{1}^{2}=x_{0}=0$.

$\left(27^{\circ}\right)$ One has $d=4$ and Type $(X)=\mathrm{A}_{4}$. The dual graph is

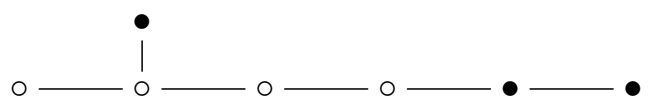

Then $X$ is not weakly minimal. The lines are cut out by $x_{0}=0$ and $x_{1}=0$.

$\left(28^{\circ}\right)$ One has $d=4$ and Type $(X)=\mathrm{A}_{3} \mathrm{~A}_{1}$. The dual graph is

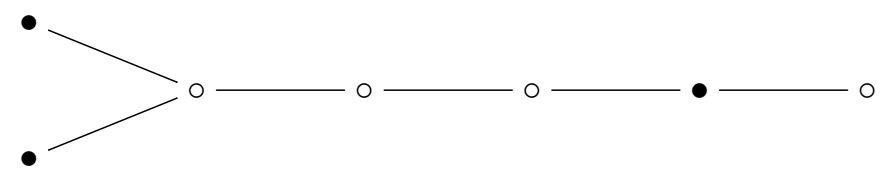

The surface $X$ is weakly minimal. The lines are $x_{2}^{2}-x_{1} x_{3}=x_{0}=0$ and $x_{1}=x_{2} \pm x_{0}^{3}=0$.

$\left(29^{\circ}\right)$ One has $d=4$ and Type $(X)=\mathrm{A}_{2} 2 \mathrm{~A}_{1}$. The dual graph is

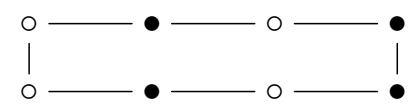

The surface $X$ is weakly minimal. The lines are cut out by $x_{2}=0$ and $x_{3}=0$. 


\section{Cheltsov and Yu. Prokhorov}

$\left(30^{\circ}\right)$ One has $d=4$ and Type $(X)=4 \mathrm{~A}_{1}$. The dual graph is

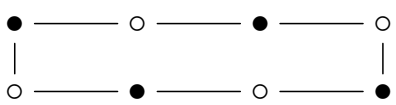

The surface $X$ is weakly minimal. The lines in $X$ are cut by $x_{4}=0$.

$\left(31^{\circ}\right)$ One has $d=4$ and Type $(X)=\mathrm{A}_{3}$ and $\#(X)=4$. The dual graph is

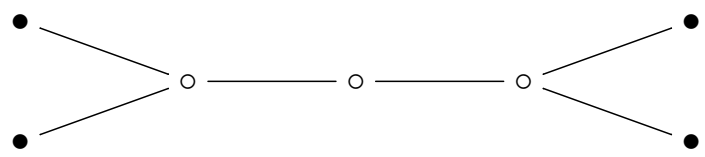

The surface $X$ is weakly minimal. The lines are $x_{2} \pm x_{1}^{2}=x_{0}=0$ and $x_{2} \pm x_{0}^{2}=x_{1}=0$.

$\left(32^{\circ}\right)$ One has $d=4$ and Type $(X)=\mathrm{A}_{3}$ and $\#(X)=5$. The dual graph is

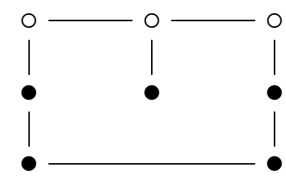

Then $X$ is not weakly minimal. The lines are cut out by $x_{0}=0$ and $x_{1}=0$.

$\left(33^{\circ}\right)$ One has $d=4$ and Type $(X)=\mathrm{A}_{2} \mathrm{~A}_{1}$. The dual graph is

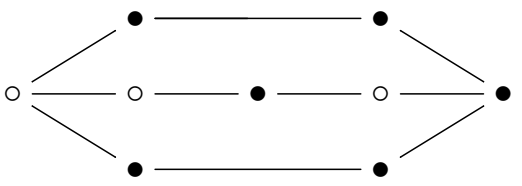

Then $X$ is not weakly minimal. The lines are cut out by $x_{1}=0$ and $x_{0}=0$.

$\left(34^{\circ}\right)$ One has $d=4$ and Type $(X)=3 \mathrm{~A}_{1}$. The dual graph is

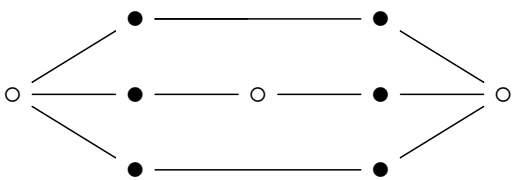

The surface $X$ is weakly minimal. The lines are cut out by $x_{2}=0$ and $x_{3}=0$.

$\left(35^{\circ}\right)$ One has $d=4$ and $\operatorname{Type}(X)=2 \mathrm{~A}_{1}$ and $\#(X)=8$. The dual graph is

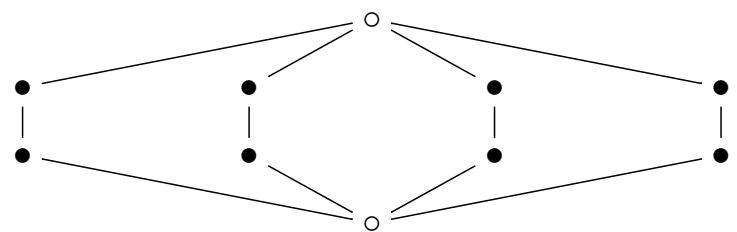

The surface $X$ is weakly minimal. The lines are cut out by $x_{2}=0$ and $x_{3}=0$.

$\left(36^{\circ}\right)$ One has $d=5$ and Type $(X)=\mathrm{A}_{4}$. The dual graph is

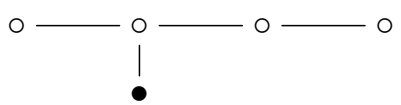

The surface $X$ is weakly minimal. The line is given by $x_{2}^{2}+x_{1}^{3}=x_{0}=0$. 


\section{Del Pezzo surfaces}

$\left(37^{\circ}\right)$ One has $d=5$ and Type $(X)=\mathrm{A}_{3}$. The dual graph is

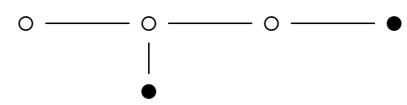

Then $X$ is weakly minimal. The lines are $x_{0}=x_{2}=0$ and $y_{1}=x_{2}=0$.

$\left(38^{\circ}\right)$ One has $d=5$ and Type $(X)=\mathrm{A}_{2} \mathrm{~A}_{1}$. The dual graph is

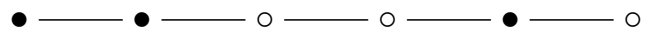

Then surface $X$ is not weakly minimal. It is given in $\mathbb{P}^{5}$ by

$$
x_{0} x_{2}=x_{1} x_{5}, \quad x_{0} x_{3}=x_{5}^{2}, \quad x_{1} x_{3}=x_{2} x_{5}, \quad x_{1} x_{4}=x_{5}^{2}, \quad x_{2} x_{4}=x_{3} x_{5} .
$$

The lines on $X$ are cut out by $x_{5}=0$.

$\left(39^{\circ}\right)$ One has $d=5$ and $\operatorname{Type}(X)=\mathrm{A}_{2}$. The dual graph is

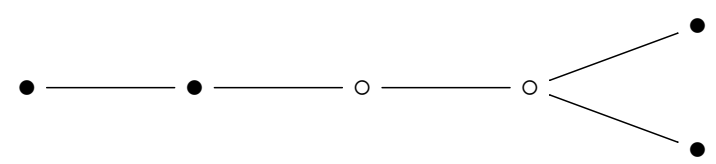

Then $X$ is not weakly minimal. The lines are cut out by $x_{0}=0$ and $x_{1}=0$.

$\left(40^{\circ}\right)$ One has $d=5$ and Type $(X)=2 \mathrm{~A}_{1}$. The dual graph is

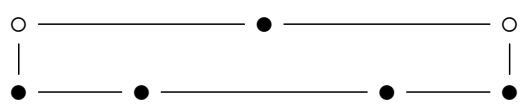

Then $X$ is not weakly minimal. The lines are cut out by $x_{0}=0, x_{1}=0$, and $x_{2}=0$.

$\left(41^{\circ}\right)$ One has $d=5$ and Type $(X)=\mathrm{A}_{1}$. The dual graph is

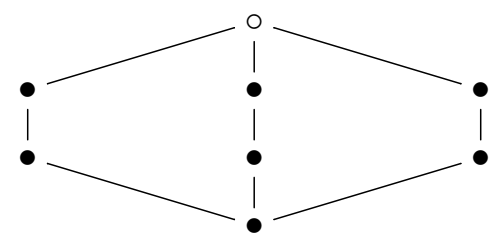

Then $X$ is not weakly minimal. The lines are cut out by $x_{0}=0, x_{1}=0, y_{0}=0$, and $y_{1}=0$.

$\left(42^{\circ}\right)$ One has $d=6$ and Type $(X)=\mathrm{A}_{2} \mathrm{~A}_{1}$. The dual graph is

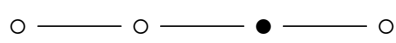

Then $X$ is weakly minimal. The line is $x_{0}=0$.

$\left(43^{\circ}\right)$ One has $d=6$ and Type $(X)=\mathrm{A}_{2}$. The dual graph is

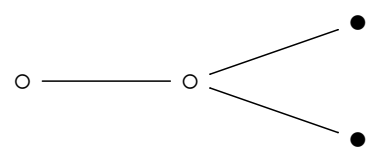

The surface $X$ is weakly minimal. The lines are $x_{0}=x_{1}=0$ and $x_{0}=x_{2}=0$.

$\left(44^{\circ}\right)$ One has $d=6$ and Type $(X)=2 \mathrm{~A}_{1}$. The dual graph is

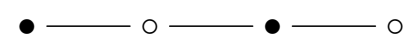

The surface $X$ is weakly minimal. The lines are cut out by $x_{2}=0$. 


\section{Cheltsov and Yu. Prokhorov}

$\left(45^{\circ}\right)$ One has $d=6$ and Type $(X)=\mathrm{A}_{1}$. The dual graph is

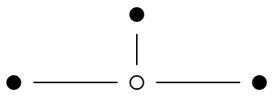

The surface $X$ is weakly minimal. The lines are cut out by $x_{2}=0$.

$\left(46^{\circ}\right)$ One has $d=6$ and Type $(X)=\mathrm{A}_{1}$ and $\#(X)=4$. The dual graph is

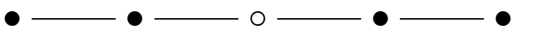

The surface $X$ is not weakly minimal. The lines are cut out by $x_{0}=0$.

$\left(47^{\circ}\right)$ One has $d=6$ and $X$ is smooth. The dual graph is

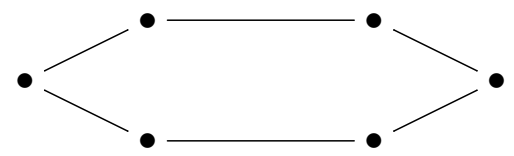

Then $X$ is not weakly minimal. The lines are cut out by $x_{0}=0, y_{0}=0$, and $z_{0}=0$.

\section{Appendix B. Del Pezzo surfaces of same homology type as $\mathbb{P}^{2}$}

In this section, we recall the classification of $\mathrm{Du}$ Val del Pezzo surfaces whose Weil divisor class group is cyclic [MZ88, Fur86, Ye02]. By Lemma 2.9(iii), each such surface except $\mathbb{P}^{2}$ and $\mathbb{P}(1,1,2)$ contains a unique line that generates its class group.

Proposition B.1. Let $X$ be a Du Val del Pezzo surface such that $\mathrm{Cl}(X) \cong \mathbb{Z}$, and let $d:=K_{X}^{2}$. Then $d \neq 7$. If $d \leqslant 6$, then there is an embedding $X \hookrightarrow \mathbb{P}(1,2,3, d)$ such that $X$ is given by

$$
\phi\left(y_{1}, y_{2}, y_{3}, y_{d}\right)=0
$$

where $\phi$ is a homogeneous polynomial of weighted degree 6 . If $2 \leqslant d \leqslant 6$, then

$$
\phi=y_{3}^{2}+y_{2}^{3}+y_{1}^{6-d} y_{d}
$$

so that $X$ is uniquely determined by its degree. If $d=1$, there are exactly two possibilities:

$$
\begin{aligned}
& \phi=y_{3}^{2}+y_{2}^{3}+y_{1}^{5} y_{d}, \\
& \phi=y_{3}^{2}+y_{2}^{3}+y_{1}^{5} y_{d}+y_{1}^{2} y_{2}^{2},
\end{aligned}
$$

which give us two non-isomorphic surfaces. The only line $L \subset X$ is cut out by $y_{1}=0$.

Proof. The proof is similar to the proof of Theorem 3.8.

Thus, if $d=9,8,6$, then $X \cong \mathbb{P}^{2}, \mathbb{P}(1,1,2), \mathbb{P}(1,2,3)$, respectively.

Remark B.4. In the notation and with the assumptions of Proposition B.1, one can show that $X \backslash L \cong \mathbb{A}^{2}$. Moreover, there is cell decomposition $X=\mathbb{A}^{2} \cup \mathbb{A}^{1} \cup \mathbb{A}^{0}$. In particular, we have

$$
H_{q}(X, \mathbb{Z}) \cong H_{q}\left(\mathbb{P}^{2}, \mathbb{Z}\right)
$$

for all $q$ (cf. [BB84]). In the case $d=1$, we can say even more: $H^{*}(X, \mathbb{Z}) \cong H^{*}\left(\mathbb{P}^{2}, \mathbb{Z}\right)$ as rings.

Remark B.5. Suppose that $X$ is a Du Val del Pezzo surface of degree 1 such that $\mathrm{Cl}(X) \cong \mathbb{Z}$. By Proposition B.1, the surface $X$ is a hypersurface in $\mathbb{P}(1,1,2,3)$ that is given

(i) either by $y_{3}^{2}+y_{2}^{3}+y_{1}^{5} y_{1}^{\prime}=0$, 


\section{Del Pezzo surfaces}

(ii) or by $y_{3}^{2}+y_{2}^{3}+y_{1}^{5} y_{1}^{\prime}+y_{1}^{2} y_{2}^{2}=0$.

These possibilities are distinguished by the collection of singular curves in the pencil $\left|-K_{X}\right|$. Indeed, in the first case, the pencil $\left|-K_{X}\right|$ contains two singular curves $y_{1}=0$ and $y_{1}^{\prime}=0$, which are both cuspidal. In the second case, it has three singular curves $y_{1}=0, y_{1}^{\prime}=0$, and $4 y_{1}+27 y_{1}^{\prime}=0$. One of them is cuspidal, and the remaining two curves are nodal.

Corollary B.6. Let $X$ be a Du Val del Pezzo surface, let $d:=K_{X}^{2}$. If $\operatorname{Cl}(X) \cong \mathbb{Z}$, then $\operatorname{Type}(X)$ is

$$
\mathrm{E}_{8}, \quad \mathrm{E}_{7}, \quad \mathrm{E}_{6}, \quad \mathrm{D}_{5}, \quad \mathrm{~A}_{4}, \quad \mathrm{~A}_{2} \mathrm{~A}_{1}, \mathrm{~A}_{1}, \varnothing
$$

in the case when $d=1,2,3,4,5,6,8,9$, respectively. Vice versa, if $\rho(X)=1$ and Type $(X)$ is one of the types in (B.7), then $\mathrm{Cl}(X) \cong \mathbb{Z}$.

Proof. The first assertion easily follows from Proposition B.1. To prove the second one, we may assume that $d \leqslant 5$ since the remaining cases are easy. Let $\mathrm{Cl}(X, P)$ be the local Weil divisor class group of the (unique) singular point $P \in X$. Then in the cases in (B.7), we have

$$
\mathrm{Cl}(X, P) \cong \mathbb{Z} / d \mathbb{Z}
$$

see, for example, [Bri68, Satz 2.11]. Therefore, for any line $L \subset X$, the divisor $d L$ is Cartier. Since $d L \sim_{\mathbb{Q}}-K_{X}$, we have $d L \sim-K_{X}$. Now, the assertion follows from Lemma 2.9(iii) and Proposition 3.4.

For every $d \in\{2, \ldots, 6\}$, let $X_{d}$ be the Du Val del Pezzo surface of degree $d$ such that $\mathrm{Cl}\left(X_{d}\right) \cong \mathbb{Z}$. As we already mentioned earlier, the surface $X_{d}$ contains exactly one line $L_{d}$ and $\mathrm{Cl}\left(X_{d}\right)=\mathbb{Z}\left[L_{d}\right]$. Take a point $P_{d} \in L_{d} \backslash \operatorname{Sing}\left(X_{d}\right)$. Let $\sigma_{d}: \widetilde{X}_{d} \rightarrow X_{d}$ be the blow-up of the point $P_{d}$, and let $\widetilde{L}_{d}$ be the proper transform on $\widetilde{X}_{d}$ of the line $L_{d}$. Then

$$
\widetilde{L}_{d}^{2}=-1+\frac{1}{d}<0
$$

and $K_{\widetilde{X}_{d}} \cdot \widetilde{L}_{d}=0$. Therefore, there exists a crepant contraction $\varphi_{d}: \widetilde{X}_{d} \rightarrow X_{d-1}^{\prime}$ of the curve $\widetilde{L}_{d}$, where $X_{d-1}^{\prime}$ is a singular Du Val del Pezzo surface such that $\mathrm{Cl}\left(X_{d-1}^{\prime}\right) \cong \mathbb{Z}$ and $K_{X_{d}^{\prime}}^{2}=K_{\widetilde{X}}^{2}=d-1$. Thus, if $d \neq 2$, then $X_{d-1}^{\prime} \cong X_{d-1}$ by Proposition B.1. If $d=2$, then $X_{1}^{\prime}$ is one of the two surfaces described in Remark B.5, so that we also let $X_{1}=X_{1}^{\prime}$. Hence, we obtain the following diagram:

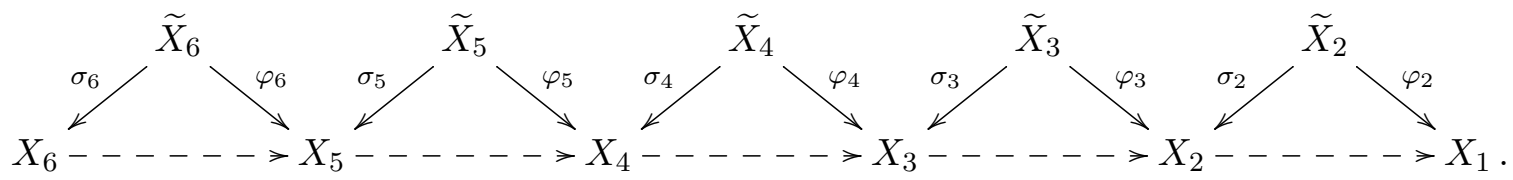

It allows us to reconstruct all surfaces in Proposition B.1 starting from $X_{6}=\mathbb{P}(1,2,3)$.

Each birational transformation $X_{d-1} \rightarrow X_{d}$ is $\operatorname{Aut}^{0}\left(X_{d-1}\right)$-equivariant, so that it gives a natural embedding $\operatorname{Aut}^{0}\left(X_{d-1}\right) \hookrightarrow \operatorname{Aut}^{0}\left(X_{d}\right)$ such that $\operatorname{Aut}^{0}\left(X_{d-1}\right)$ is just the stabilizer of the point $P_{d}$. Moreover, there following two assertions hold:

- If $d \geqslant 3$, then $\operatorname{Aut}^{0}\left(X_{d}\right)$ transitively acts on $L_{d} \backslash \operatorname{Sing}\left(X_{d}\right)$.

- If $d=2$, then $\operatorname{Aut}^{0}\left(X_{2}\right) \cong \mathbb{G}_{\mathrm{m}}$ has two orbits in $L_{2} \backslash \operatorname{Sing}\left(X_{2}\right)$, an open orbit and a closed orbit that consists of a single point, which explains the two possibilities in Remark B.5.

The construction also allows us to compute $\operatorname{Aut}^{0}(X)$ in the cases $1^{\circ}, 5^{\circ}, 14^{\circ}, 24^{\circ}, 36^{\circ}$ of Table 3 . 


\section{Cheltsov and Yu. Prokhorov}

Corollary B.8. Let $X$ be a $D u$ Val del Pezzo surface such that $\mathrm{Cl}(X) \cong \mathbb{Z}$, and let $d:=K_{X}^{2}$. Then the group $\operatorname{Aut}^{0}(X)$ is isomorphic to

$$
\mathbb{B}_{3}, \quad \mathbb{B}_{2} \times \mathbb{G}_{\mathrm{m}}, \quad \mathbb{G}_{\mathrm{a}}^{2} \rtimes \mathbb{G}_{\mathrm{m}}, \quad \mathbb{G}_{\mathrm{a}} \rtimes_{(3)} \mathbb{G}_{\mathrm{m}}, \quad \mathbb{G}_{\mathrm{m}}
$$

in the case when $d=6,5,4,3,2$, respectively. If $d=1$, then $\operatorname{Aut}^{0}(X) \cong \mathbb{G}_{\mathrm{m}}$ if $X$ is given by (B.2), and $\operatorname{Aut}^{0}(X)=\{1\}$ if $X$ is given by (B.3).

\section{ACKNOWLEDGEMENTS}

The authors would like to thank the anonymous referee for much valuable advice that helped to improve this paper.

\section{REFERENCES}

ABHX21 J. Alper, H. Blum, D. Halpern-Leistner, and C. Xu, Reductivity of the automorphism group of K-polystable Fano varieties, Invent. Math. 222 (2020), 995-1032; doi:10.1007/s00222-02000987-2.

ADHL15 I. Arzhantsev, U. Derenthal, J. Hausen, and A. Laface, Cox rings, Cambridge Stud. Adv. Math., vol. 144 (Cambridge Univ. Press, Cambridge, 2015).

BB84 D. Bindschadler and L. Brenton, On singular 4-manifolds of the homology type of $\mathbf{C P}^{2}$, J. Math. Kyoto Univ. 24 (1984), no. 1, 67-81; doi:10.1215/kjm/1250521385.

Bre80 L. Brenton, On singular complex surfaces with negative canonical bundle, with applications to singular compactifications of $\mathbf{C}^{2}$ and to 3-dimensional rational singularities, Math. Ann. 248 (1980), no. 2, 117-124; doi:10.1007/BF01421952.

Bri68 E. Brieskorn, Rationale Singularitäten komplexer Flächen, Invent. Math. 4 (1968), 336-358; doi:10.1007/BF01425318.

BW79 J.W. Bruce and C.T.C. Wall, On the classification of cubic surfaces, J. London Math. Soc. 19 (1979), no. 2, 245-256; doi:10.1112/jlms/s2-19.2.245.

CT88 D.F. Coray and M. A. Tsfasman, Arithmetic on singular Del Pezzo surfaces, Proc. London Math. Soc. 57 (1988), no. 1, 25-87; doi:10.1112/plms/s3-57.1.25.

Dem80 M. Demazure, Surfaces de del Pezzo. I. II. III. IV. V, Sémin. sur les singularités des surfaces, Cent. Math. Ec. Polytech. (Palaiseau 1976-1977), Lecture Notes in Math., vol. 777 (Springer, Berlin, 1980), 21-69; doi:10.1007/BFb0084119.

DI09 I. V. Dolgachev and V. A. Iskovskikh, Finite subgroups of the plane Cremona group, in Algebra, Arithmetic, and Geometry: in Honor of Yu. I. Manin, Vol. I, Progr. Math., vol. 269 (Birkhäuser Boston, Boston, MA, 2009), 443-548; doi:10.1007/978-0-8176-4745-2_11.

DL10 U. Derenthal and D. Loughran, Singular del Pezzo surfaces that are equivariant compactifications, J. Math. Sci. 171 (2010), 714-724; doi:10.1007/s10958-010-0174-9.

DL15___ Equivariant compactifications of two-dimensional algebraic groups, Proc. Edinb. Math. Soc. 58 (2015), no. 1, 149-168; doi:10.1017/S001309151400042X.

Dol12 I. V. Dolgachev, Classical algebraic geometry: a modern view (Cambridge Univ. Press, Cambridge, 2012); doi:10.1017/CB09781139084437.

DuV34 P. Du Val, On isolated singularities of surfaces which do not affect the conditions of adjunction. I-III, Proc. Camb. Philos. Soc. 30 (1934), no. 4, 453-459, 460-465, 483-491; doi:10.1017/ S030500410001269X, doi:10.1017/S0305004100012706, doi:10.1017/S030500410001272X.

Fur86 M. Furushima, Singular del Pezzo surfaces and analytic compactifications of 3-dimensional complex affine space $\mathbf{C}^{3}$, Nagoya Math. J. 104 (1986), 1-28; doi:10.1017/S0027763000022649. 


\section{Del Pezzo surfaces}

Her14 E. Herppich, On Fano varieties with torus action of complexity 1, Proc. Edinb. Math. Soc. 57 (2014), no. 3, 737-753; doi:10.1017/S0013091513000710.

HHS11 J. Hausen, E. Herppich, and H. Süß, Multigraded factorial rings and Fano varieties with torus action, Doc. Math. 16 (2011), 71-109.

Hui79 C. M. Hui, Plane quartic curves, Ph.D. Thesis, University of Liverpool, 1979, available at https://www.liverpool.ac.uk/ pjgiblin/papers/hui-thesis.pdf.

HW81 F. Hidaka and K. Watanabe, Normal Gorenstein surfaces with ample anti-canonical divisor, Tokyo J. Math. 4 (1981), no. 2, 319-330; doi:10.3836/tjm/1270215157.

KST85 B. E. Kunyavskii, A. N. Skorobogatov, and M. A. Tsfasman, The combinatorics and geometry of del Pezzo surfaces of degree four, Russian Math. Surveys 40 (1985), no. 6, 131-132; doi: 10.1070/RM1985v040n06ABEH003713.

Mat57 Y. Matsushima, Sur la structure du groupe d'homéomorphismes analytiques d'une certaine variété kählérienne, Nagoya Math. J. 11 (1957), 145-150; doi:10.1017/S0027763000002026.

Mor85 D. R. Morrison, The birational geometry of surfaces with rational double points, Math. Ann. 271 (1985), no. 3, 415-438; doi:10.1007/BF01456077.

MS20 G. Martin and C. Stadlmayr, Weak del Pezzo surfaces with global vector fields, 2020, arXiv: 2007.03665.

MZ88 M. Miyanishi and D.-Q. Zhang, Gorenstein log del Pezzo surfaces of rank one, J. Algebra 118 (1988), no. 1, 63-84; doi:10.1016/0021-8693(88)90048-8.

OSS16 Y. Odaka, C. Spotti, and S. Sun, Compact moduli spaces of del Pezzo surfaces and KählerEinstein metrics, J. Differential Geom. 102 (2016), no. 1, 127-172; doi:10.4310/jdg/ 1452002879.

Pro01 Yu. G. Prokhorov, Lectures on complements on log surfaces, MSJ Mem., vol. 10 (Math. Soc. Japan, Tokyo, 2001).

PR00 B. Poonen and F. Rodriguez-Villegas, Lattice polygons and the number 12, Amer. Math. Monthly 107 (2000), no. 3, 238-250; doi:10.2307/2589316.

Rei87 M. Reid, Young person's guide to canonical singularities, Algebraic Geometry, Bowdoin, 1985 (Brunswick, Maine, 1985), Proc. Sympos. Pure Math., vol. 46 (Amer. Math. Soc., Providence, RI, 1987), 345-414.

Sak10 Y. Sakamaki, Automorphism groups on normal singular cubic surfaces with no parameters, Trans. Amer. Math. Soc. 362 (2010), no. 5, 2641-2666; doi : 10.1090/S0002-9947-09-05023-5

Wal95 C. T. C. Wall, Geometry of quartic curves, Math. Proc. Cambridge Philos. Soc. 117 (1995), no. 3, 415-423; doi:10.1017/S0305004100073266.

Ye02 Q. Ye, On Gorenstein log del Pezzo surfaces, Japan. J. Math. (N.S.) 28 (2002), no. 1, 87-136; doi:10.4099/math1924.28.87.

Ivan Cheltsov I.Cheltsov@ed.ac.uk

University of Edinburgh, Edinburgh, UK

National Research University Higher School of Economics, Moscow, Russia

Yuri Prokhorov prokhoro@mi-ras.ru

Steklov Mathematical Institute, Moscow, Russia

National Research University Higher School of Economics, Moscow, Russia 